\title{
Least Ambiguous Set-Valued Classifiers with Bounded Error Levels
}

\author{
Mauricio Sadinle ${ }^{1}$, Jing Lei ${ }^{2}$, and Larry Wasserman ${ }^{2 *}$ \\ ${ }^{1}$ Department of Biostatistics, University of Washington, Seattle, WA, USA \\ ${ }^{2}$ Department of Statistics, Carnegie Mellon University, Pittsburgh, PA, USA
}

\begin{abstract}
In most classification tasks there are observations that are ambiguous and therefore difficult to correctly label. Set-valued classifiers output sets of plausible labels rather than a single label, thereby giving a more appropriate and informative treatment to the labeling of ambiguous instances. We introduce a framework for multiclass set-valued classification, where the classifiers guarantee user-defined levels of coverage or confidence (the probability that the true label is contained in the set) while minimizing the ambiguity (the expected size of the output). We first derive oracle classifiers assuming the true distribution to be known. We show that the oracle classifiers are obtained from level sets of the functions that define the conditional probability of each class. Then we develop estimators with good asymptotic and finite sample properties. The proposed estimators build on existing single-label classifiers. The optimal classifier can sometimes output the empty set, but we provide two solutions to fix this issue that are suitable for various practical needs.
\end{abstract}

Keywords: Ambiguous observation; Bayes classifier; Reject option; Multiclass classification; Non-deterministic classifier; Oracle classifier.

* Mauricio Sadinle is Assistant Professor in the Department of Biostatistics, University of Washington, Seattle, WA 98195 (e-mail: msadinle@uw.edu). Jing Lei is Assistant Professor, Department of Statistics, Carnegie Mellon University, Pittsburgh, PA 15213 (e-mail: jinglei@andrew.cmu.edu). Larry Wasserman is Professor, Department of Statistics and Machine Learning Department, Carnegie Mellon University (e-mail: larry@stat.cmu.edu). Mauricio Sadinle was supported by NSF grants SES11-30706 and SES-11-31897. Jing Lei's research was partially supported by NSF grants DMS-1407771 and DMS-1553884. 


\section{Introduction}

Let $(X, Y) \in \mathcal{X} \times \mathcal{Y}$ be a random vector with unknown distribution $\mathbb{P}$. Here $\mathcal{X}$, typically a subset of $\mathbb{R}^{d}$, is a feature space, and $\mathcal{Y}=\{1, \ldots, K\}$ is the label space. In the traditional multiclass classification problem the goal is to label each point $x \in \mathcal{X}$ with the appropriate class label $y \in \mathcal{Y}$ in a way that is coherent with $\mathbb{P}$. Unlike standard classifiers, which output a single label for each point in $\mathcal{X}$, our goal is to construct a set-valued classifier that assigns a set of plausible labels to each point in $\mathcal{X}$, while assuming that each sample point truly belongs to a single class. Our motivation comes from the fact that in most classification tasks there are ambiguous observations whose true class is difficult to determine, yet traditional classifiers are forced to output single labels. We argue that assigning sets of plausible labels provides a better treatment to such instances and therefore leads to a more informative approach to classification. While there already exist approaches to setvalued classification, we derive set-valued classifiers that guarantee lower bounds on the probability of containing the true label while minimizing the average number of assigned labels.

A set-valued classifier is a function $\mathbf{H}: \mathcal{X} \mapsto 2^{\mathcal{Y}}$, or in other words, $\mathbf{H}(x)$ is a subset of $\{1, \ldots, K\}$ for each $x \in \mathcal{X}$. We denote the joint distribution of $(X, Y)$ on $\mathcal{X} \times \mathcal{Y}$ by $\mathbb{P}$. Having a set-valued output allows us to guarantee levels of confidence in our predictions. We consider two types of coverage guarantees:

$$
\begin{array}{ll}
\text { Total } & \mathbb{P}\{Y \in \mathbf{H}(X)\} \geq 1-\alpha, \\
\text { Class-Specific } & \mathbb{P}\{Y \in \mathbf{H}(X) \mid Y=y\} \geq 1-\alpha_{y} \text { for all } y \in \mathcal{Y},
\end{array}
$$

where we refer to $\alpha\left(\left\{\alpha_{y}\right\}_{y=1}^{K}\right)$ as the error level(s), and to $1-\alpha\left(\left\{1-\alpha_{y}\right\}_{y=1}^{K}\right)$ as the coverage or confidence level(s). 
Once we fix the desired levels of confidence there are further properties that we want our classifiers to have. In particular, we want a classifier that assigns multiple plausible labels to ambiguous observations, but that does so no more than needed. We therefore would like a classifier $\mathbf{H}$ with minimal ambiguity, which we define as

$$
\mathbb{A}(\mathbf{H})=\mathbb{E}\{|\mathbf{H}(X)|\},
$$

where $|\cdot|$ is the number of points in a set.

In Section 2 we provide a full characterization of the optimal set-valued classifiers, which we refer to as LABEL (least ambiguous with bounded error levels). These optimal classifiers correspond to level sets of the conditional probability functions $p(y \mid x)$, that is, they have the form $\left\{y: p(y \mid x) \geq t_{y}\right\}$ for some thresholds $\left\{t_{y}\right\}_{y=1}^{K}$, where $p(y \mid x) \equiv \mathbb{P}(Y=y \mid X=x)$. A potentially undesirable property of the optimal classifiers is that they may lead to empty predictions, that is, $\mathbf{H}(x)=\emptyset$ for some points $x \in \mathcal{X}$, especially when the required coverage is low. We call $\mathcal{N}=\{x: \mathbf{H}(x)=\emptyset\}$ the null region. This region arises because minimizing ambiguity can favor making $\mathbf{H}(x)$ empty, and because some classes may be relatively well separated with respect to the coverage requirements. We provide solutions to this issue in Section 3 .

We consider generic plug-in estimators in Section 4, together with a technique called inductive conformal inference (Papadopoulos et al., 2002, Vovk et al., 2005; Shafer and Vovk, 2008; Vovk, 2013) or split-conformal inference (Lei et al., 2014), which we use to adjust the classifiers to have finite sample, distribution-free coverage under essentially no conditions. We will also see in Section 4, that all of our analyses carry through even if we let the number of classes $K$ increase with $n$ as long as $K \equiv K_{n}=o(\sqrt{n / \log n})$. In Section 5 we present data examples that show the advantages of LABEL classifiers. 


\section{$1.1 \quad$ Related work}

Classifiers that output possibly more than one label are known as set-valued classifiers (Grycko, 1993) or non-deterministic classifiers (del Coz et al., 2009). In another related framework called classification with reject option (Chow, 1970; Herbei and Wegkamp, 2006; Bartlett and Wegkamp, 2008; Yuan and Wegkamp, 2010; Ramaswamy et al. 2015), a classifier may reject to output a definitive class label if the uncertainty is high. Set-valued classification contains this framework as a special case, as one can view the "reject to classify" option as outputting the entire set of possible labels. These methods for set-valued classification generally follow the idea of minimizing a modified loss function. For example, Herbei and Wegkamp (2006) assigns a constant loss $\rho \in(0,1 / 2)$ for the output "reject," while del Coz et al. (2009) defines the loss function as a weighted combination of precision and recall in an information retrieval framework. Certain components of such modified loss functions, such as the loss of the output "reject" and the weight used to combine precision and recall, lack direct practical meaning and may be hard to choose for practitioners.

Another line of related work is Vovk et al. (2005) and Shafer and Vovk (2008), who introduced a method called "conformal prediction" that yields set-valued classifiers with finite sample confidence guarantees. In fact, we will show that many of our results are related to those of Vovk et al. (2014, 2016) for conformal prediction. Lei et al. (2014, 2013), Lei and Wasserman (2014), and Lei (2014) studied the conformal approach from the point of view of statistical optimality in the unsupervised, regression and binary classification cases, respectively. We make use of conformal ideas in Sections 3 and 5. Recently, Denis and Hebiri (2015) used asymptotic plug-in methods to derive classification confidence sets in the binary case. They control a different quantity, namely, the coverage conditional on $\mathbf{H}(X)$ having a single element. Finally, we notice 
that although it would seem appealing to aim at controlling the conditional coverage $\mathbb{P}\{Y \in \mathbf{H}(X) \mid X=x\} \geq 1-\alpha$, for all $x \in \mathcal{X}$, which Vovk (2013) calls "object validity," Lemma 1 of Lei and Wasserman (2014) unfortunately implies that if $X$ is

continuous and $\widehat{\mathbf{H}}$ has distribution-free conditional validity, then $\widehat{\mathbf{H}}$ is trivial, meaning that $\widehat{\mathbf{H}}(x)=\{1, \ldots, K\}$.

At this point it is important to point out that our contributions do not belong to the literature on "multi-label" or "multi-output" classification (see, e.g. Tsoumakas and Katakis, 2007; Zhang and Zhou, 2007), since in that context each sample point actually has multiple co-occurring characteristics that one wants to jointly predict, such as the presence/absence of multiple diseases in a hospital patient. A classifier in that context naturally assigns multiple labels to each sample point — one label associated to each possibly co-occurring characteristic. In this article, on the contrary, each sample unit truly belongs to only one out of $K$ mutually exclusive classes, but we use a set-valued output to represent the plausible classes for each sample unit.

\subsection{Contributions}

Our framework improves and generalizes the ideas of Lei (2014) to the case of $K \geq 2$ classes, where $K$ can even grow with the sample size. For binary classification, Lei (2014) proposed to find two prediction regions $C_{y} \subset \mathcal{X}, y=1,2$, as the solution to minimizing $\mathbb{P}\left\{X \in C_{1} \cap C_{2}\right\}$ subject to $\mathbb{P}\left\{X \in C_{y} \mid Y=y\right\} \geq 1-\alpha_{y}, y=1,2$, and $C_{1} \cup C_{2}=\mathcal{X}$. A first difficulty of that approach is that, as stated, the problem cannot be generalized to the multiclass case in a simple manner, and so our extension is technically non-trivial. Most importantly, although Lei (2014)'s construction seems ideal, the interaction of the problem constraints may lead to multiple solutions, some of which do not provide a meaningful treatment of ambiguous observations. If we 
drop the restriction $C_{1} \cup C_{2}=\mathcal{X}$, the solution to this optimization problem can correspond to regions that do not naturally overlap, thereby leading to a region of empty predictions (null region). Imposing the constraint $C_{1} \cup C_{2}=\mathcal{X}$ in such situations leads to multiple solutions, one of which is to fill in the null region with an arbitrary class, which is indeed the solution provided by Lei (2014). That solution, however, conceals the characteristics of the classification task at hand: the null region arises because the classes are relatively well separated with respect to the coverage requirements. In other words, in certain classification tasks we may be able to afford higher confidence levels than the ones initially required. Furthermore, arbitrarily filling in the null region defeats our goal of giving a proper treatment to ambiguous instances, as we illustrate throughout the article, and it is particularly clear in the application to the zip code data in Section 5.5. With multiple classes, arbitrarily filling in the null region no longer corresponds to an optimal solution after imposing the constraint $\cup_{y=1}^{K} C_{y}=\mathcal{X}$ (the excess risk of this approach is characterized in Theorem 8 . We therefore provide alternative solutions that give a more appropriate handling of ambiguous instances (Section 3). Some of our new arguments provide further insights to the problem and lead to significantly more straightforward characterization and estimation of the optimal classifiers. For example, our Theorems 1 and 6 and lemma 5 . and their proofs, are very different from the results presented by Lei (2014).

\section{Optimal procedures}

Our discussion will focus on the case $\mathcal{X} \subseteq \mathbb{R}^{d}$. Let $\mathbb{P}$ denote the joint distribution of $(X, Y)$ on $\mathcal{X} \times \mathcal{Y}$. In this section we derive LABEL classifiers assuming that $\mathbb{P}$ is known, but in Section 4 we present different estimation procedures. We assume $\mathbb{P}$ to be absolutely continuous with respect to $\nu(x, y)=\nu_{X}(x) \nu_{Y}(y)$ where $\nu_{X}$ is the 
Lebesgue measure and $\nu_{Y}$ is the counting measure. Let $p$ be the density of $\mathbb{P}$ with respect to $\nu$. Throughout the article, we denote $p(x \mid y) \equiv p_{y}(x) \equiv p(x \mid Y=y)$, where $p(\cdot \mid Y=y)$ is a density of the conditional distribution of $X$ given $Y=y$, which is assumed to be positive on $\mathcal{X}$ for each $y=1, \ldots, K$. We let $\pi_{y} \equiv \mathbb{P}(Y=y)$ denote the marginal class probabilities and denote $p(y \mid x) \equiv \mathbb{P}(Y=y \mid X=x)$. We assume $\pi_{y}>0$ for all $y$. A set-valued classifier $\mathbf{H}$ can be represented by a collection of sets

$$
C_{y}=\{x \in \mathcal{X}: y \in \mathbf{H}(x)\}, \text { for } y=1, \ldots, K .
$$

Then, $\mathbf{H}(x)=\left\{y: x \in C_{y}\right\}$. Finally, with a little abuse of notation, we can also define $\mathbf{H}$ as a subset of $\mathcal{X} \times \mathcal{Y}$ :

$$
\mathbf{H}=\{(x, y): y \in \mathbf{H}(x)\} .
$$

Note that $\mathbf{H}(x)$ is the $x$-section of $\mathbf{H}$ and $C_{y}$ is the $y$-section of $\mathbf{H}$.

\subsection{Total coverage}

We start by considering the problem of minimizing the ambiguity subject to an upper bound $\alpha$ on the total probability of an error, that is:

$$
\min _{\mathbf{H}} \mathbb{E}\{|\mathbf{H}(X)|\} \quad \text { subject to } \quad \mathbb{P}\{Y \notin \mathbf{H}(X)\} \leq \alpha .
$$

Theorem 1. Assume that $p(Y \mid X)$ does not have a point mass at its $\alpha$ quantile, denoted $t_{\alpha}$. The classifier that optimizes (1) is given by

$$
\mathbf{H}_{\alpha}^{*}=\left\{(x, y): p(y \mid x) \geq t_{\alpha}\right\} .
$$

This optimal classifier can be written as $\mathbf{H}_{\alpha}^{*}(x)=\left\{y: p(y \mid x) \geq t_{\alpha}\right\}$. 
Theorem 1 is a consequence of Lemma 2 by choosing $f=p(x, y)$ and $g=p(x)$. If $p(Y \mid X)$ has a point mass at its $\alpha$ quantile, define $t_{\alpha}=\sup [t: \mathbb{P}\{p(Y \mid X) \geq$ $t\} \geq 1-\alpha]$, and $D_{\alpha}=\left\{(x, y): p(y \mid x)=t_{\alpha}\right\}$. Then we must have $\mathbb{P}\left(D_{\alpha}\right)>0$. If $X$ has a continuous distribution then we can choose a subset $D^{\prime} \subseteq D_{\alpha}$ and let $\mathbf{H}_{\alpha}^{*}=\left\{(x, y): p(y \mid x)>t_{\alpha}\right\} \cup D^{\prime}$, with $\mathbb{P}\left(\mathbf{H}_{\alpha}^{*}\right)=1-\alpha$. If $X$ is discrete, such a subset $D^{\prime}$ may not always exist, but we can use a randomized rule on $D_{\alpha}$ as in the original Neyman-Pearson Lemma. In the rest of this paper we will avoid this complication by assuming the distribution of $p(Y \mid X)$ being continuous at $t_{\alpha}$.

Lemma 2 (Neyman-Pearson). Let $f$ and $g$ be two nonnegative measurable functions, then the optimizer of the problem

$$
\min _{C} \int_{C} g \text { subject to } \int_{C} f \geq 1-\alpha
$$

is given by $C=\{f / g \geq t\}$ if there exists $t$ such that $\int_{f \geq t g} f=1-\alpha$.

The problem given in expression (1) is equivalent to the one studied by Lei (2014) for the special case of $K=2$, but we do not impose the restriction $C_{1} \cup C_{2}=\mathcal{X}$. This constraint leads to optimal arbitrary solutions that may not be meaningful for handling ambiguous instances, as argued in Section 1.2. Instead in Section 3 we will provide more principled solutions when the initial classification regions do not cover the whole feature space. Moreover, the characterization provided in Theorem 1 is much simpler than that given in Lei (2014). Recently, Vovk et al. (2016) proved an equivalent result in the context of conformal prediction, where their Theorem 1 is similar to our Theorem 1 .

Although it seems reasonable to work with procedures that control the total probability of an error, in some circumstances this approach may lead to unsatisfactory classifiers. In particular, when one of the classes is much more prevalent than the others, the probability of properly labeling an element of the smaller classes may be quite low, 

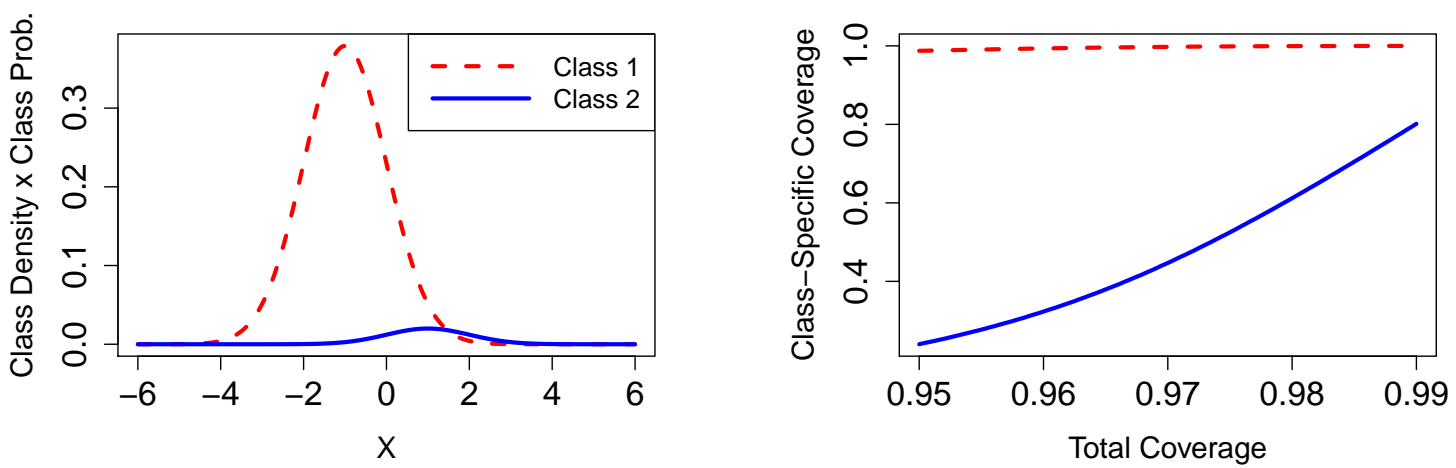

Figure 1: This figure shows the two classes from Example 3. Left: class-specific densities. Right: class-specific coverage as a function of total coverage.

and it decreases as the probability of the largest class increases. We illustrate this behavior with the following example.

Example 3. Consider $\mathcal{X}=\mathbb{R}, \mathcal{Y}=\{1,2\}, \mathbb{P}(Y=1)=0.95$, and the distributions $(X \mid Y=y)$ being normal with means $\mu_{1}=-1$ and $\mu_{2}=1$, and standard deviations equal to 1. In Figure 1 we show the densities of the two classes and the specific coverage of each class as a function of the total coverage. We can see that the probability of correctly labeling an element of class 2 can be quite low, whereas we would correctly label elements of class 1 with probability almost equal to 1.

The previous example indicates that a more appropriate approach should control the coverage of each class, as we show in the next section.

\subsection{Class-specific coverage}

We now derive LABEL classifiers when controlling the individual coverage of each class. We consider the following problem:

$$
\min _{\mathbf{H}} \mathbb{E}\{|\mathbf{H}(X)|\} \quad \text { subject to } \quad \mathbb{P}\{Y \notin \mathbf{H}(X) \mid Y=y\} \leq \alpha_{y} \quad \text { for all } y
$$


for pre-specified error levels $\alpha_{y}, y=1, \ldots, K$. Moreover, as we shall see, LABEL classifiers also minimize the probabilities of incorrect label assignments $\mathbb{P}\{y \in \mathbf{H}(X) \mid Y \neq y\}$ for all $y$.

Remark 4. The ambiguity of a set-valued classifier $\mathbf{H}$ can be expressed as

$$
\mathbb{E}\{|\mathbf{H}(X)|\}=\sum_{y=1}^{K} \mathbb{P}\{y \in \mathbf{H}(X)\} .
$$

Lemma 5. If a set-valued classifier $\mathbf{H}$ minimizes the probabilities of incorrect label assignments $\mathbb{P}\{y \in \mathbf{H}(X) \mid Y \neq y\}$ for all $y \in\{1, \ldots, K\}$ among all classifiers that have certain error levels $\left\{\alpha_{y}\right\}_{y=1}^{K}$, then it also minimizes the ambiguity.

Theorem 6. Given a set of error levels $\left\{\alpha_{y}\right\}_{y=1}^{K}$, the set-valued classifier induced by the sets $C_{y}=\left\{x: p(y \mid x) \geq t_{y}\right\}$, with $t_{y}$ chosen so that $\mathbb{P}\left(C_{y} \mid Y=y\right)=1-\alpha_{y}$, simultaneously minimizes the probabilities of incorrect label assignments for all $y$ and the ambiguity.

Theorem 6 tells us how to find LABEL classifiers when controlling the error probability $\alpha_{y}$ for each class. A referee pointed out that a similar result had been presented by Vovk et al. (2014) in the context of conformal prediction. The solution in Theorem 6 may lead to empty predictions, that is, there may exist a region of $\mathcal{X}$ where $\mathbf{H}(x)=\emptyset$. This phenomenon can also occur when controlling the total probability of an error as in Section 2.1. The presence of this null region occurs when the upper bounds on the error levels are large, when the classes are well separated, or in practice it could happen if we have sample points that are anomalies, that is, points that do not fit any of the existing classes. In any case, we shall propose principled solutions to cover this region, but we first illustrate the procedure given by Theorem 6 with an example and defer the discussion on null regions to Section 3 .

Example 7. We consider an example with $\mathcal{X}=\mathbb{R}^{2}, \mathcal{Y}=\{1,2,3\}, \mathbb{P}(Y=y)=1 / 3$ for all $y$, and the distributions $(X \mid Y=y)$ being bivariate normal with means $\mu_{1}=$ 

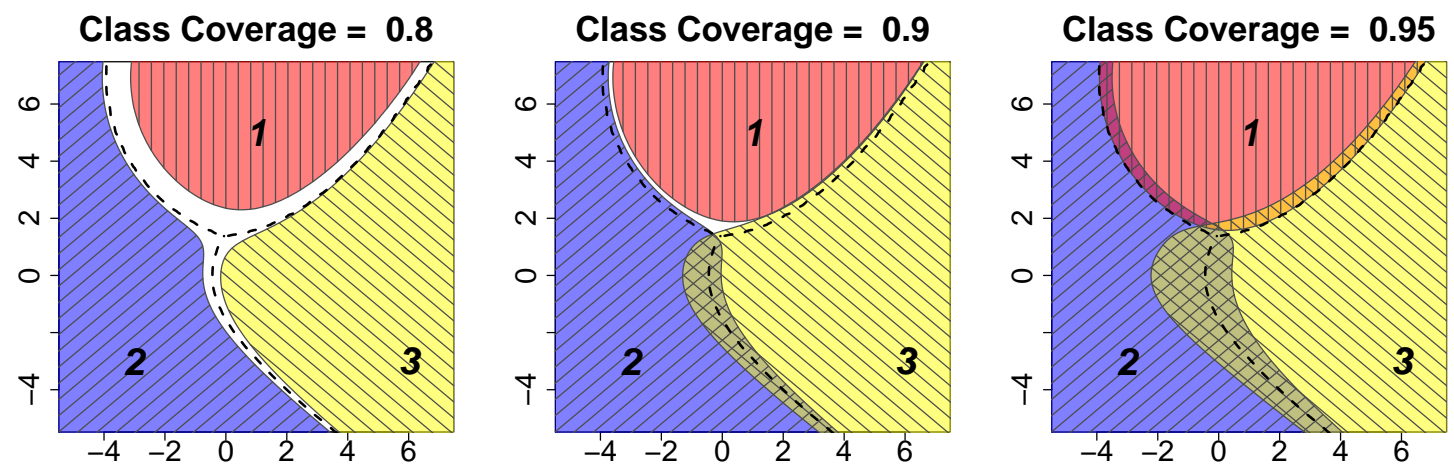

Figure 2: This figure shows the three classes from Example 7. For reference, the black dashed lines denote the boundaries of the Bayes classifier's regions. Left: Optimal classifier when $\alpha_{y}=0.2$ for each class. In this case there is a null region (white) corresponding to $\mathbf{H}(x)=\emptyset$. Middle: Optimal classifier when $\alpha_{y}=0.1$ for each class. The null region is smaller and an ambiguous region has appeared. Right: Optimal classifier when $\alpha_{y}=0.05$ for each class. The null region is gone but the ambiguity has further increased.

$(0,3.5), \mu_{2}=(-2,0)$ and $\mu_{3}=(0,2)$, and covariance matrices specified as $\Sigma_{1}=I_{2}$, $\Sigma_{2}=2 I_{2}$, and $\Sigma_{3}=\operatorname{diag}(5,1)$, with $I_{p}$ representing the $p \times p$ identity matrix, and diag representing a diagonal matrix. In Figure 2 we show the classification regions $C_{y}$ obtained from Theorem 6 for different values of class-specific coverage $1-\alpha_{y}$, with $\alpha_{y}=\alpha$ for all $y, \alpha=0.2,0.1,0.05$. We can see that when the required class-specific coverage is not large enough the procedure can lead to a null region. On the other hand, the null region disappears when the class-specific coverage becomes large and ambiguous classification regions arise as overlaps of the $C_{y}$ regions. 


\section{Dealing with null regions}

Given a set-valued classification rule $\mathbf{H}$, the null region is $\mathcal{N}=\mathcal{N}(\mathbf{H})=\{x: \mathbf{H}(x)=$ $\emptyset\}$. We present two methods for eliminating these regions of empty predictions.

The first approach, called "filling with baseline classifier" simply uses a fixed baseline classifier to fill in the null region. This method is fast and simple, but it may not properly capture some ambiguous areas of the feature space. Nevertheless, it provides a simple solution if controlling the nominal error levels is the only concern.

The second method, called "accretive completion," gradually grows the optimal classifier by decreasing the class thresholds $t_{y}$ that define the classes $C_{y}$ (see Theorem 6), while minimizing the increments in ambiguity, until the null region is eliminated. This approach is more principled and more aligned with the motivation of our framework as it can possibly identify further ambiguous areas inside the null region. We demonstrate this property with the examples presented in Section 5. We recommend this method when thorough detection of ambiguous regions is of concern.

\subsection{Approach I: Filling with a baseline classifier}

A simple solution to the problem of the null region is to complete the set-valued classifier with a given baseline classifier, such as the Bayes classifier. Let $h(\cdot)$ be a simple classifier such that $|h(x)|=1$ for all $x$, and define

$$
\mathbf{H}^{\dagger}(x)= \begin{cases}\mathbf{H}(x) & \text { if } \mathbf{H}(x) \neq \emptyset \\ h(x) & \text { if } \mathbf{H}(x)=\emptyset .\end{cases}
$$


To justify this method, we start from the following optimization problem that explicitly avoids null region:

$$
\min _{\mathbf{H}} \mathbb{E}\{|\mathbf{H}(X)|\}, \quad \text { subject to } \mathbf{H}(x) \neq \emptyset, \forall x, \quad \mathbb{P}\left(C_{y} \mid y\right) \geq 1-\alpha_{y}, \forall y
$$

Problem (3) is hard to solve, in general, but the following theorem says that $\mathbf{H}^{\dagger}$ is close to optimal when the null region is small.

Theorem 8 (Excess risk bound of $\mathbf{H}^{\dagger}$ ). Let $\widetilde{\mathbb{A}}$ be the optimal value of problem (3), $\mathbf{H}^{*}$ a solution to (2), and $\mathbf{H}^{\dagger}$ a classifier such that $\left|\mathbf{H}^{\dagger}(x)\right|=1$ if $x \in \mathcal{N}\left(\mathbf{H}^{*}\right)$ and $\mathbf{H}^{\dagger}(x)=\mathbf{H}^{*}(x)$ if $x \notin \mathcal{N}\left(\mathbf{H}^{*}\right)$ then $\widetilde{\mathbb{A}} \leq \mathbb{A}\left(\mathbf{H}^{\dagger}\right) \leq \widetilde{\mathbb{A}}+\mathbb{P}\left\{\mathcal{N}\left(\mathbf{H}^{*}\right)\right\}$.

The case of binary classification deserves special attention, given that in that case $\mathbf{H}^{\dagger}$ always achieves the optimal value $\widetilde{\mathbb{A}}$ of problem (3). If the initial LABEL classifier $\mathbf{H}$ leads to a null region, we argue that the regions $C_{1}$ and $C_{2}$ have to be disjoint. For $C_{1}$ and $C_{2}$ to have an overlap there would have to exist $x \in \mathcal{X}$ such that $p(1 \mid x) \geq t_{1}$ and $p(2 \mid x) \geq t_{2}$, which would imply $1=p(1 \mid x)+p(2 \mid x) \geq t_{1}+t_{2}$. But on the other hand, for all elements $x$ in the null region $p(1 \mid x)<t_{1}$ and $p(2 \mid x)<t_{2}$, which implies $1=p(1 \mid x)+p(2 \mid x)<t_{1}+t_{2}$. We conclude that the existence of a null region in the binary case implies $C_{1} \cap C_{2}=\emptyset$. In that case, the optimal value $\widetilde{\mathbb{A}}=1$, which is achieved by $\mathbf{H}^{\dagger}$. Finally, if the initial LABEL classifier $\mathbf{H}$ does not lead to a null region then $\mathbf{H}^{\dagger}=\mathbf{H}$, which achieves $\widetilde{\mathbb{A}}$.

It is important to point out that problem (3) can have multiple solutions, some of which may not necessarily be meaningful when our goal is to properly detect and deal with ambiguous regions, as we explain in Example 9. It is also worth noticing that the excess risk bound of Theorem 8 also characterizes the procedure that fills in the null region with an arbitrary class. This motivates the usage of a different approach, as presented in the next subsection.

Example 9. Consider the scenario given by Example 7 with 0.8 class-specific cov- 
erage. In this case the regions $C_{y}^{\prime}$ that are the optimal solution of problem (2) are all disjoint and do not cover the whole feature space (left panel of Figure 2). The null region includes cases that are truly ambiguous, that is, cases where either $\mathbb{P}(Y=y \mid x) \approx 1 / 3$ for $y=1,2,3$, or $\mathbb{P}(Y=y \mid x) \approx 1 / 2$ for two values of $Y$, and therefore assigning a single label to such cases would not allow us to properly handle their ambiguity. Now, notice that by adding the constraint $\bigcup_{y=1}^{K} C_{y}=\mathcal{X}$ to problem (2) we have that the minimum value of the ambiguity is 1 . Given that the regions $C_{y}^{\prime}$ already achieve the desired levels of coverage, any partition $\left\{C_{y}^{*}\right\}_{y=1}^{K}$ of $\mathcal{X}$ such that $C_{y}^{\prime} \subseteq C_{y}^{*}$ represents an optimal classifier. Interestingly, this includes the option of filling in the null region with an arbitrary class, that is, define $C_{y}^{*}=C_{y}^{\prime}$ for all $y \neq y_{0}$ and $C_{y_{0}}^{*}=C_{y_{0}}^{\prime} \cup\left(\bigcup_{y=1}^{K} C_{y}^{\prime}\right)^{c}$ for some arbitrary $y_{0} \in\{1, \ldots, K\}$. We conclude that the problem given in Expression (3) may lead to solutions that are not appropriate for handling ambiguity in classification.

Remark 10. One can also reduce the chance of having a null region by assigning the same ambiguity loss to the empty set and sets with cardinality one. That is, for $\mathbf{H}(x) \subseteq \mathcal{Y}$, we define the loss function $\ell\{\mathbf{H}(x)\}=\max \{|\mathbf{H}(x)|-1,0\}$, and look for classifiers $\mathbf{H}$ minimizing $\mathbb{E}[\ell\{\mathbf{H}(X)\}]$, subject to coverage requirements. Theorem 3 of Vovk et al. (2016) provides a characterization, under total coverage, of the optimal classifier among all idealized conformal classifiers (classifiers obtained by thresholding some function $g(x, y))$. We note that the loss function $\ell$ does not explicitly eliminate null regions, which is possible to occur if the optimal value is 0 .

\subsection{Approach II: Accretive completion}

If a set-valued classifier $\mathbf{H}$ has a non-empty null region $\mathcal{N}(\mathbf{H})=\{x: \mathbf{H}(x)=\emptyset\}$, we call $\mathbf{H}$ inadmissible, otherwise we call $\mathbf{H}$ admissible. Given $\boldsymbol{t}=\left(t_{1}, \ldots, t_{K}\right)$, denote 
$\mathbf{H}_{\boldsymbol{t}}=\left\{(x, y): p(y \mid x) \geq t_{y}\right\}$. In Theorem 6 we showed that, for any $\left\{\alpha_{y}\right\}_{y=1}^{K}$, the solution to the problem (2) is $\mathbf{H}_{\boldsymbol{t}}$ with $\boldsymbol{t}$ chosen such that $\mathbb{P}\left\{Y \in \mathbf{H}_{\boldsymbol{t}}(X) \mid Y=y\right\}=$ $1-\alpha_{y}$. Under this solution the inadmissibility of $\mathbf{H}_{\boldsymbol{t}}$ implies $\sum_{y} t_{y}>1$. To see this, note that $\mathbf{H}_{\boldsymbol{t}}(x)=\emptyset$ implies that $p(y \mid x)<t_{y}$ for all $y$. Hence, $1=\sum_{y} p(y \mid x)<\sum_{y} t_{y}$. Therefore, a sufficient condition for $\mathbf{H}_{t}$ to be admissible is that $\sum_{y=1}^{K} t_{y} \leq 1$.

Now suppose that given nominal error levels $\left\{\alpha_{y}\right\}_{y=1}^{K}$, the solution $\mathbf{H}_{\boldsymbol{t}^{(0)}}$, with $\boldsymbol{t}^{(0)}=$ $\left(t_{1}^{(0)}, \ldots, t_{K}^{(0)}\right)$, to problem (2) given by Theorem 6 is inadmissible. We propose to search for a set of thresholds $\left\{t_{y}\right\}_{y=1}^{K}$ that has the lowest ambiguity and guarantees admissibility as well as nominal coverage of $\mathbf{H}_{\boldsymbol{t}}$ :

$$
\min _{\boldsymbol{t}} \mathbb{E}\left\{\left|\mathbf{H}_{\boldsymbol{t}}(X)\right|\right\} \quad \text { subject to } t_{y} \leq t_{y}^{(0)}, \forall y ; \quad \sum_{y} t_{y} \leq 1
$$

We can proceed in a greedy way to approximate the solution of this problem. The idea is to decrease the thresholds $t_{y}$, one at a time, in such a way that at each step we achieve the lowest increment in ambiguity. The detailed procedure is given in Algorithm 1. Notice that for a threshold vector $\boldsymbol{t}$ the ambiguity function can be written as $\mathbb{A}(\boldsymbol{t})=\sum_{y=1}^{K} \mathbb{P}\left\{p(y \mid X) \geq t_{y}\right\}$.

Example 11. To build the intuition for the accretive completion procedure consider the first panel of Figure 3 (nominal coverage 0.95 for each class). We can see that by increasing the coverage of region 1 many points in the null region would be covered by region 1 alone. On the other hand if we wanted to increase the coverage of region 3 we would not cover many points in the null region but we would increase the ambiguity. This indicates that we can approximate the solution to the problem by decreasing the thresholds of the different regions at different rates.

In Figure 3 the second panel shows the solution given by the accretive completion procedure which leads to ambiguity of 1.028. We can see that the null region was covered mostly by class 1 since $t_{1}$ went from 0.99 to 0.206, whereas the other thresholds 


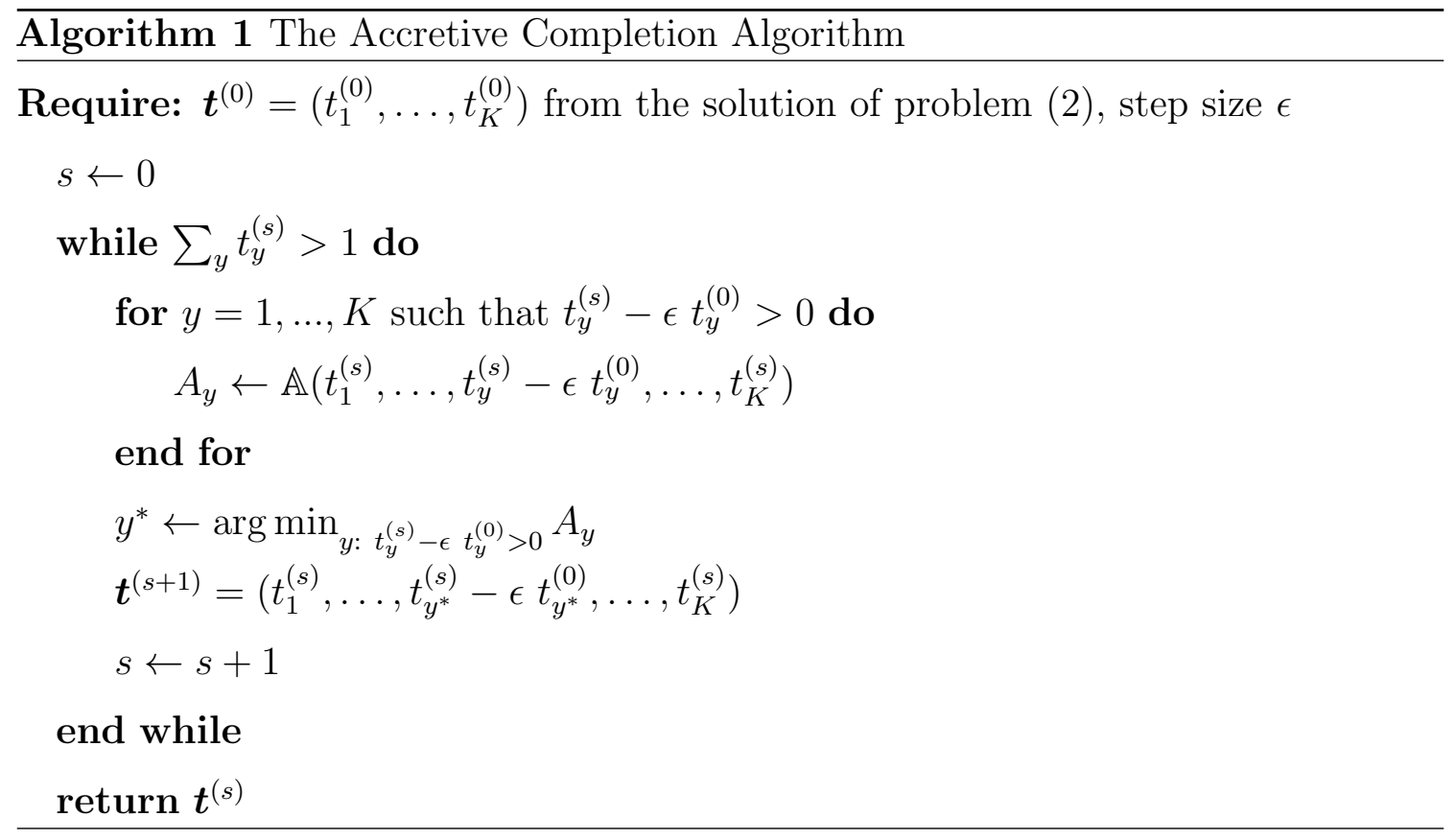

did not decrease much.

Although the following remark is obvious from the construction of the accretive completion algorithm, it emphasizes a desirable property of the method.

Remark 12. Let $\mathbf{H}^{+}$be the classifier obtained from the accretive completion procedure and $\left\{\alpha_{y}^{+}\right\}_{y=1}^{K}$ its final error levels. Since the algorithm never increases the thresholds $t_{y}$, we necessarily have $\alpha_{y}^{+} \leq \alpha_{y}$, for all $y$, where $\alpha_{y}$ is the initial error level. Also, $\mathbf{H}^{+}$is the solution to the problem in expression (2) for error levels $\left\{\alpha_{y}^{+}\right\}_{y=1}^{K}$.

Here we note that accretive completion is not necessary in the binary classification case, since the approach of filling with a baseline classifier in the previous subsection already leads to a classifier with the lowest ambiguity, and if we use the Bayes classifier to fill-in the null region we already obtain a classifier with the form $\mathbf{H}_{t}=\left\{(x, y): p(y \mid x) \geq t_{y}\right\}$, with $t_{1}=1-t_{2}$.

It is also possible to use a similar strategy for growing the total coverage classifier. 

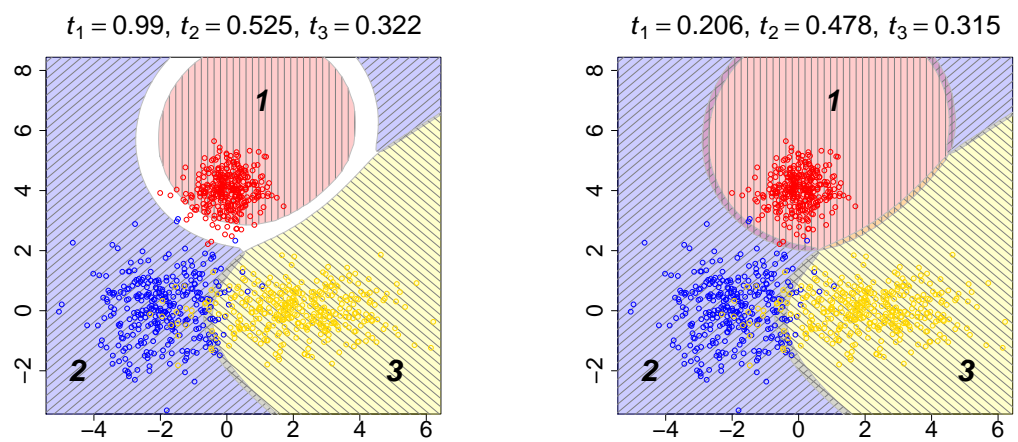

Figure 3: Left: original solution with class coverage of 0.95. Right: solution of accretive completion algorithm, ambiguity 1.028. Sample points are drawn to indicate where the probability mass is concentrated.

Recall that, in that case, $\mathbf{H}(x)=\{y: p(y \mid x) \geq t\}$, for a single threshold $t$. Growing the classifier corresponds to reducing $t$ until there are no null regions. It is easy to see that this happens when $t=1 / K$. To have the desired coverage, we also need $t \leq t_{\alpha}$, where $t_{\alpha}$ is the threshold given by Theorem 1. We summarize this as a lemma.

Lemma 13. The classifier of the form $\mathbf{H}_{\boldsymbol{t}}=\{(x, y): p(y \mid x) \geq t\}$ that minimizes the ambiguity, with total coverage at least $1-\alpha$ and empty null-region is given by $\mathbf{H}(x)=\left\{y: p(y \mid x) \geq(1 / K) \wedge t_{\alpha}\right\}$, where $t_{\alpha}$ is specified in Theorem 1 .

This lemma suggests that when growing the classifier it is better to use class-specific coverage as above, especially when $K$ is large.

\section{Estimation and finite sample adjustment}

Now we consider estimating LABEL classifiers using independent draws $\left(X_{i}, Y_{i}\right), i=$ $1, \ldots, n$, from $\mathbb{P}$. We first study plug-in methods which asymptotically mimic the optimal procedures with rates of convergence under standard regularity conditions. 
Then we combine these asymptotically optimal procedures with split-conformal inference to achieve a distribution-free, finite sample coverage guarantee.

\subsection{The plug-in approach}

The optimal classifiers in the previous sections are level sets of $p(y \mid x)$. There are two things we need to estimate for each $y=1, \ldots, K$ : the regression function $p(y \mid x)$ and the threshold $t_{y}$ (when controlling total coverage there is a single threshold $t_{\alpha}$ ).

The initial estimator of $p(y \mid x)$. Any conventional estimator of $p(y \mid x)$ can be plugged into the optimal classifiers. This could be a direct estimate of $p(y \mid x)$ or we could estimate $\pi_{y}$ and $p_{y}(x)$ and then use $p(y \mid x)=\pi_{y} p_{y}(x) / \sum_{\ell} \pi_{\ell} p_{\ell}(x)$. Here are some specific examples:

(a) $k$ Nearest Neighbors $(k N N)$. For any $x$, let $d_{k}(x)$ be the distance from $x$ to its $k$ th nearest neighbor. Define $\widehat{p}(y \mid x)=k^{-1} \sum_{i=1}^{n} I\left(Y_{i}=y\right) I\left\{\left\|X_{i}-x\right\| \leq d_{k}(x)\right\}$. We ignore cases where several neighbors exist at distance $d_{k}(x)$, since that occurs with probability zero in our set-up. We will demonstrate our framework with this estimator in the zip code data example in Section 5.

(b) Local polynomial estimator: $p(y \mid x)$ is estimated as the regression function of $I(Y=y)$ given $X=x$ using the standard local polynomial estimator Tsybakov, 2009). This covers the kernel estimator as a special case: $\widehat{p}(y \mid x) \propto \sum_{i=1}^{n} I\left(Y_{i}=\right.$ y) $K_{h}\left(x-X_{i}\right)$, where $K_{h}$ is a kernel with bandwidth $h$.

(c) Regularized multinomial logistic regression: $\widehat{p}(y \mid x)=\exp \left(\widehat{\theta}_{y}^{T} x\right) /\left\{1+\exp \left(\widehat{\theta}_{y}^{T} x\right)\right\}$, where $\widehat{\theta}_{y}$ is a possibly penalized logistic regression coefficient of $I(Y=y)$ on $X$.

Level sets. Having estimated $p(y \mid x)$ we can determine the cut-off point for the level set according to the target coverage. 
Case 1: Total coverage. For the total coverage, define

$$
\widehat{\operatorname{Cov}}(t)=\frac{1}{n} \sum_{i=1}^{n} I\left\{\widehat{p}\left(Y_{i} \mid X_{i}\right) \geq t\right\} .
$$

Let

$$
\widehat{t}=\sup \{t: \widehat{\operatorname{Cov}}(t) \geq 1-\alpha\}=\max _{i}\left[\widehat{p}\left(Y_{i} \mid X_{i}\right): \widehat{\operatorname{Cov}}\left\{\widehat{p}\left(Y_{i} \mid X_{i}\right)\right\} \geq 1-\alpha\right] .
$$

Finally, we take $\widehat{\mathbf{H}}(x)=\{y: \widehat{p}(y \mid x) \geq \widehat{t}\}$.

Case 2: Class-specific coverage. For the class-specific case, let $\boldsymbol{t}=\left(t_{1}, \ldots, t_{K}\right)$ and define $\widehat{C}_{y}=\left\{x: \widehat{p}(y \mid x) \geq t_{y}\right\}$. The plug-in estimate of the coverage for class $y$ is

$$
\widehat{\operatorname{Cov}}_{y}\left(t_{y}\right)=\frac{\sum_{i=1}^{n} I\left(X_{i} \in \widehat{C}_{y}\right) I\left(Y_{i}=y\right)}{\sum_{i=1}^{n} I\left(Y_{i}=y\right)} .
$$

Let

$$
\widehat{t}_{y}=\sup \left\{t: \widehat{\operatorname{Cov}}_{y}\left(t_{y}\right) \geq 1-\alpha_{y}\right\}=\max _{i: Y_{i}=y}\left[\widehat{p}\left(Y_{i} \mid X_{i}\right): \widehat{\operatorname{Cov}}_{y}\left\{\widehat{p}\left(Y_{i} \mid X_{i}\right)\right\} \geq 1-\alpha_{y}\right]
$$

Finally, we take $\widehat{\mathbf{H}}(x)=\left\{y: \widehat{p}(y \mid x) \geq \widehat{t}_{y}\right\}$.

Null region. If the initial $\widehat{C}_{y}$ 's lead to a null region, we can use a plug-in version of accretive completion (Algorithm 1) to cover the whole feature space. To do this, we replace in Algorithm 1 each $t_{y}^{(0)}$ by $\widehat{t}_{y}$ obtained above, and $\mathbb{A}(\boldsymbol{t})$ by $\widehat{\mathbb{A}}(\boldsymbol{t})=$ $n^{-1} \sum_{i=1}^{n} \sum_{y=1}^{K} I\left\{\widehat{p}\left(y \mid X_{i}\right) \geq t_{y}\right\}$.

\subsection{Asymptotic properties}

There are two main assumptions for the development of asymptotic properties of the plug-in level set estimator. The first one is concerned with the accuracy of $\widehat{p}$. Assume $\widehat{p}$ satisfies

$$
\mathbb{P}\left\{\sup _{x}\left|\widehat{p}_{n}(y \mid x)-p(y \mid x)\right| \geq \epsilon_{n}\right\} \leq \delta_{n}, \quad \forall 1 \leq y \leq K .
$$


All conventional estimators mentioned in the previous subsection satisfy this sup-norm consistency under appropriate conditions.

(a) $k$ NN classifiers: when $p(y \mid x)$ is Lipschitz on $x$, and the distribution of $X$ has intrinsic dimension $d$, then (4) is satisfied by $k \mathrm{NN}$ classifiers with $\delta_{n}=n^{-1}$ and $\epsilon_{n} \asymp(\log n / n)^{1 /(2+d)}$ when $d \leq 2\left((\log n / n)^{1 /(2 d)}\right.$ for $\left.d \geq 3\right)$ Devroye, 1978).

(b) Local polynomial estimators: when $p(y \mid x)$ is $\beta$-Hölder smooth, and $\mathcal{X}$ has dimension $d$, then (4) holds with $\epsilon_{n} \asymp(\log n / n)^{\beta /(2 \beta+d)}, \delta_{n} \asymp n^{-1}$ for local polynomial estimator of order $\beta$ (Stone, 1982; Audibert and Tsybakov, 2007; Lei, 2014).

(c) Logistic regression estimators: in the case where $d$ is large, if the logistic regression model $p(y \mid x)=\exp \left(\beta_{y}^{T} x\right) /\left\{1+\exp \left(\beta_{y}^{T} x\right)\right\}$ holds and $\widehat{\beta}_{y}$ is estimated with appropriately chosen $\ell_{1}$ penalty, then (4) holds with $\epsilon_{n} \asymp(\log d / n)^{1 / 4}$ and $\delta_{n} \asymp\left(d^{-1}+\sqrt{\log d / n}\left\|\beta_{y}\right\|_{0}\right)$, provided that the minimum eigenvalue of $\mathbb{E}\left(X X^{T}\right)$ is bounded away from 0 (van de Geer, 2008), where $\|\cdot\|_{0}$ denotes the number of non-zero entries of a vector.

The second assumption is on the density of $p(y \mid X)$ near the cut-off value. For $1 \leq y \leq K$, let $G_{y}$ be the distribution function of $p(y \mid X)$ with $X$ distributed as $\mathbb{P}_{y}$, the conditional distribution of $X$ given $Y=y$. Let $t_{y}=G_{y}^{-1}\left(\alpha_{y}\right)$ be the ideal cut-off value for $p(y \mid x)$. Let $G=\sum_{y=1}^{K} \pi_{y} G_{y}$ be the distribution of $p(Y \mid X)$, with $(X, Y)$ distributed as $\mathbb{P}$.

The density condition is, for some constants $\gamma, c_{1}, c_{2}, s_{0}$,

$$
c_{1}|s|^{\gamma} \leq\left|G_{y}\left(t_{y}+s\right)-G_{y}\left(t_{y}\right)\right| \leq c_{2}|s|^{\gamma}, \forall s \in\left[-s_{0}, s_{0}\right], 1 \leq y \leq K .
$$

The corresponding condition for the total coverage is

$$
c_{1}|s|^{\gamma} \leq\left|G_{y}(t+s)-G_{y}(t)\right| \leq c_{2}|s|^{\gamma}, \forall s \in\left[-s_{0}, s_{0}\right], 1 \leq y \leq K .
$$


The difference is that the threshold $t$ is common for all classes.

Theorem 14. Suppose that (4) and (5) hold, then there exists a constant c such that with probability $1-K \delta_{n}-n^{-1}$ the plug-in class-specific classifier $\left\{\widehat{C}_{y}\right\}_{y=1}^{K}$ satisfies

$$
\mathbb{P}_{y}\left(\widehat{C}_{y} \triangle C_{y}^{*}\right) \leq c\left(\epsilon_{n}^{\gamma}+\sqrt{\frac{\log n}{n}}\right),
$$

where $\mathbb{P}_{y}$ is the conditional distribution of $X$ given $Y=y$. If (6) holds instead of (5), then there exists a constant $c$ such that with probability $1-K \delta_{n}-n^{-1}$ the total coverage classifier $\widehat{\mathbf{H}}$ satisfies

$$
\mathbb{P}\left(\widehat{\mathbf{H}} \triangle \mathbf{H}^{*}\right) \leq c\left(\epsilon_{n}^{\gamma}+K \sqrt{\frac{\log n}{n}}\right),
$$

where $\mathbb{P}$ is the joint distribution of $(X, Y)$, and $\mathbf{H}^{*}$ is the corresponding oracle classifier.

Remark 15. Suppose we let $K \equiv K_{n}$ increase with $n$. Then $\mathbb{P}\left(\widehat{\mathbf{H}} \triangle \mathbf{H}^{*}\right)$ will still go to 0 as long as $K_{n}=o(\sqrt{n / \log n})$. Thus, our results include the case where the number of classes increases with $n$ as long as it does not increase too fast.

\subsection{Finite sample coverage via split-conformal inference}

Using a method called split-conformal inference (Lei et al., 2014) (also known as inductive conformal inference in Papadopoulos et al., 2002; Vovk, 2013), we can guarantee distribution-free, finite sample validity of the classifiers.

Total coverage. The method splits the data in two halves indexed by $\mathcal{I}_{1}$ and $\mathcal{I}_{2}$. The first half is used to estimate the conditional probabilities $\widehat{p}(y \mid x)$, and the second half is used to estimate the distribution of $\widehat{p}(Y \mid X),(X, Y) \sim \mathbb{P}$. Consider augmenting the second half with a hypothetical sample point $\left(X^{*}, Y^{*}\right)$. Under the assumption that 
this new point is drawn independently from $\mathbb{P}$, the values $\left\{\widehat{p}\left(Y^{*} \mid X^{*}\right)\right\} \cup\left\{\widehat{p}\left(Y_{i} \mid X_{i}\right)\right\}_{i \in \mathcal{I}_{1}}$ are exchangeable, and so

$$
\sigma\left(X^{*}, Y^{*}\right) \equiv \frac{1}{\left|\mathcal{I}_{2}\right|+1}\left[\sum_{j \in \mathcal{I}_{2}} I\left\{\widehat{p}\left(Y_{j} \mid X_{j}\right) \leq \widehat{p}\left(Y^{*} \mid X^{*}\right)\right\}+1\right]
$$

is uniformly distributed over $\left\{1 /\left(\left|\mathcal{I}_{2}\right|+1\right), 2 /\left(\left|\mathcal{I}_{2}\right|+1\right), \ldots, 1\right\}$. Therefore, $\sigma\left(X^{*}, Y^{*}\right)$ can be used to test the hypothesis $H_{0}:\left(X^{*}, Y^{*}\right) \sim \mathbb{P}$. Given that under $H_{0}$, $\mathbb{P}_{\sigma}\left\{\sigma\left(X^{*}, Y^{*}\right) \leq \alpha\right\} \leq \alpha$, for $\alpha \in[0,1]$, we have $\mathbb{P}_{\sigma}\left\{\sigma\left(X^{*}, Y^{*}\right)>\alpha\right\} \geq 1-\alpha$, and hence any realization of $\left(X^{*}, Y^{*}\right)$ such that

$$
\sum_{j \in \mathcal{I}_{2}} I\left\{\widehat{p}\left(Y_{j} \mid X_{j}\right) \leq \widehat{p}\left(Y^{*} \mid X^{*}\right)\right\}>\alpha\left(\left|\mathcal{I}_{2}\right|+1\right)-1
$$

would not be rejected as being drawn from $\mathbb{P}$. We then need to find

$$
\widehat{t}=\min _{i \in \mathcal{I}_{2}}\left\{\widehat{p}\left(Y_{i} \mid X_{i}\right): \sum_{j \in \mathcal{I}_{2}} I\left\{\widehat{p}\left(Y_{j} \mid X_{j}\right) \leq \widehat{p}\left(Y_{i} \mid X_{i}\right)\right\}>\left(\left|\mathcal{I}_{2}\right|+1\right) \alpha-1\right\},
$$

and the set of values in $\mathcal{X} \times \mathcal{Y}$ that would not be rejected is given by $\{(x, y): \widehat{p}(y \mid x) \geq$ $\widehat{t}\} \equiv \widehat{\mathbf{H}}$. It is thus clear that for any distribution, and any $n, \mathbb{P}_{*}\{Y \in \widehat{\mathbf{H}}(X)\} \geq 1-\alpha$, where $\mathbb{P}_{*}$ is the distribution of the augmented second half of the sample.

Class-specific coverage. To guarantee finite sample, distribution-free validity for class-specific coverage we need to apply the previous method separately to each class. More specifically, as before, we split the data in two halves indexed by $\mathcal{I}_{1}$ and $\mathcal{I}_{2}$, and we use the first half to estimate the conditional probabilities $\widehat{p}(y \mid x)$. We partition the second half into $K$ groups corresponding to each class, indexed by $\mathcal{I}_{2, y}=\left\{i \in \mathcal{I}_{2}: Y_{i}=y\right\}, y \in 1, \ldots, K$. Consider augmenting $\left\{X_{i}\right\}_{i \in \mathcal{I}_{2, y}}$ with a hypothetical $X^{*}$, under the hypothesis $H_{0}: X^{*} \sim \mathbb{P}_{y}$. If we follow analogous arguments as for total coverage, we obtain that if we find

$$
\widehat{t_{y}}=\min _{i \in \mathcal{I}_{2, y}}\left\{\widehat{p}\left(y \mid X_{i}\right): \sum_{j \in \mathcal{I}_{2, y}} I\left\{\widehat{p}\left(y \mid X_{j}\right) \leq \widehat{p}\left(y \mid X_{i}\right)\right\}>\left(\left|\mathcal{I}_{2, y}\right|+1\right) \alpha_{y}-1\right\}
$$


then the classifier $\widehat{\mathbf{H}}$ obtained from the sets $\widehat{C}_{y}=\left\{x: \widehat{p}(y \mid x) \geq \widehat{t}_{y}\right\}$ has classspecific finite sample coverage $\mathbb{P}_{*}\{Y \in \widehat{\mathbf{H}}(X) \mid Y=y\} \geq 1-\alpha_{y}, y=1, \ldots, K$, where $\mathbb{P}_{*}(\cdot \mid Y=y)$ represents the joint distribution of the augmented $\left\{X_{i}\right\}_{i \in \mathcal{I}_{2, y}}$ sample. Vovk (2013) calls this guarantee "label validity."

The accretive completion (Algorithm 1) can be used easily with the split-conformal estimator because one can just apply it to the second half of the data, while taking $\widehat{p}(y \mid x)$ as an external input. To do this, we replace in Algorithm 1 each $t_{y}^{(0)}$ by $\widehat{t}_{y}$ obtained from (7), and $\mathbb{A}(\boldsymbol{t})$ by $\widehat{\mathbb{A}}(\boldsymbol{t})=\left|\mathcal{I}_{2}\right|^{-1} \sum_{i \in \mathcal{I}_{2}} \sum_{y=1}^{K} I\left\{\widehat{p}\left(y \mid X_{i}\right) \geq t_{y}\right\}$.

The theoretical results developed in Theorem 14 also apply to the split-conformal estimator, because the key technical ingredients in the assumption and the proof, such as the sup norm consistency of $\widehat{p}$ and the empirical distribution of $p(y \mid X)$, apply to the split-conformal case.

Theorem 16. Let $\left\{\widehat{C}_{y}\right\}_{y=1}^{K}$ be the split-conformal classifier constructed from a plug-in classifier $\widehat{p}(y \mid x)$. Then the results of Theorem 14 hold for $\left\{\widehat{C}_{y}\right\}_{y=1}^{K}$ under the same conditions, and the classifier has correct distribution-free finite sample coverage.

\section{Examples and Comparisons}

In this section we present four examples that illustrate LABEL classification. In three of these (Sections 5.2, 5.4, and 5.5), the sample sizes are large so we implement the split conformal method. In Section 5.3 the sample size is small so we do not split the data and only study the in-sample performance. We also demonstrate LABEL classifiers using different base estimators, including kernel regression (Section 5.2), $k \mathrm{NN}$ (Sections 5.2 and 5.5), multi-class logistic regression (Section 5.3), and sparse multi-class logistic regression (Section 5.4. We also compare our results with those 
obtained using the framework of classification with reject option (Chow, 1970; Herbei and Wegkamp, 2006), mentioned in Section 1.1, where a classifier may reject to output a class label if the correct label is highly uncertain. The Supplementary Materials contain additional simulation studies comparing the two frameworks.

\subsection{Comparing to classification with reject option}

A classifier with reject option (CWR) is a function $h_{R}: \mathcal{X} \mapsto\{1, \ldots, K, R\}$, with $R$ representing the reject option, which is typically assigned to sample units that are difficult to classify. The optimal classifier is derived by minimizing the expected value of the loss function:

$$
\ell(y, \widetilde{y})=\left\{\begin{array}{l}
1, \text { if } \widetilde{y} \neq y, \widetilde{y} \neq R \\
\rho, \text { if } \widetilde{y}=R \\
0, \text { if } \widetilde{y}=y .
\end{array}\right.
$$

Here $\rho$ should in principle be fixed as the loss of $R$ expressed as a fraction of the loss of a missclassification. From this loss function the optimal classifier is

$$
h_{R}^{*}(x)=\left\{\begin{array}{l}
\underset{y \in\{1, \ldots, K\}}{\arg \max } p(y \mid x), \text { if } \exists y: p(y \mid x) \geq 1-\rho \\
R, \text { otherwise; }
\end{array}\right.
$$

from which we can define the set-valued classifier

$$
\mathbf{H}_{R}^{*}(x)=\left\{\begin{array}{l}
\{y\}, \text { if } h_{R}^{*}(x)=y \\
\{1, \ldots, K\}, \text { if } h_{R}^{*}(x)=R .
\end{array}\right.
$$

LABEL classification and classification with reject option are fundamentally different frameworks as they control different quantities. Nevertheless, we use LABEL classifiers 
under total coverage for our comparisons. Given $\widehat{p}(y \mid x)$, a CWR is completely determined by the loss $\rho$ associated to $R$, and therefore to make our comparisons as fair as possible we choose $\rho$ so that $\mathbf{H}_{R}^{*}$ in (8) gets the same total coverage of our LABEL classifiers. Note however that this is not always possible, since with $\rho>1-1 / K$ the CWR never outputs $R$.

\subsection{A synthetic nonparametric example}

We illustrate LABEL classification using a simulated two dimensional dataset of size $n=2000$ obtained from the distribution presented in Example 11. We estimate $p(y \mid x)$ nonparametrically using $\widehat{p}(y \mid x)=\widehat{p}_{y}(x) \widehat{\pi}_{y} / \sum_{l} \widehat{p}_{l}(x) \widehat{\pi}_{l}$, where $\widehat{\pi}_{y}=\sum_{i} I\left(Y_{i}=y\right) / n$ and $\widehat{p}_{y}(x)$ is a kernel density estimator with bandwidth chosen by Silverman's rule (Silverman, 1986). We use split-conformal prediction as described in Section 4.3.

The top left plot of Figure 4 shows the LABEL classifier regions $\widehat{C}_{y}(y=1,2,3)$ fixing the total coverage at $98 \%$, which leads to an ambiguity of 1.083 . In this case we did not obtain a null region, but this may not be the case with other datasets. The overlap of the $\widehat{C}_{y}$ regions represent the areas of the feature space where more than one label is deemed plausible according to this LABEL classifier. As we had previously argued, controlling the total coverage can lead to imbalance in terms of the specific coverage of each class. In this case the classes 1, 2, and 3 have estimated specific coverage of $99.7 \%, 97.2 \%$ and $97.2 \%$, respectively.

In the top row and second column of Figure 4 we present the results from CWR choosing $\rho$ so that the set-valued classifier gets $98 \%$ total coverage. The ambiguity of this classifier is 1.164, and the estimated class coverages are $99.7 \%, 97.2 \%$ and 96.9\%. CWR leads to three regions associated to the single labels 1,2 , and 3 , and one general area where the classifier rejects to classify. From comparing the first two 


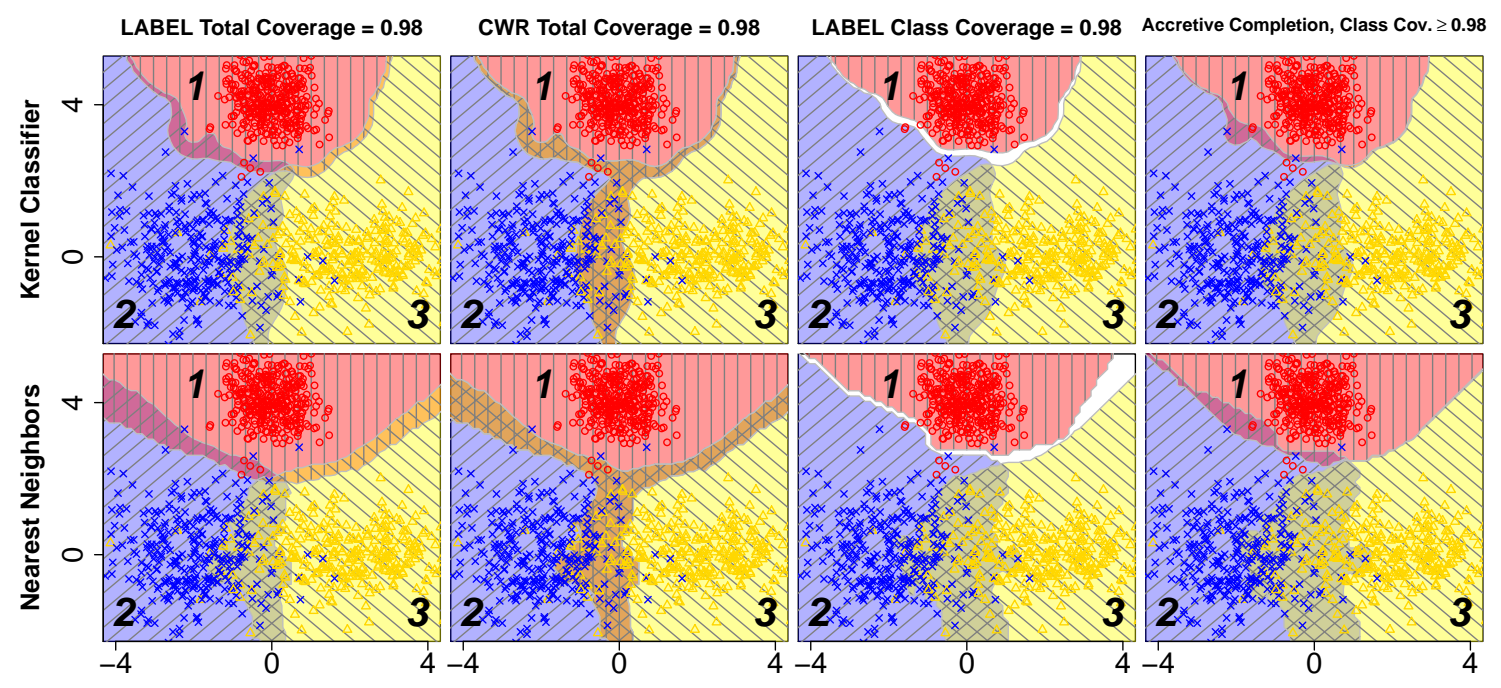

Figure 4: Prediction regions under different set-valued classifiers (columns) using baseline Kernel and nearest neighbors classifiers (rows). The true data labels are presented as $\circ$ for $Y=1, \times$ for $Y=2$, and $\triangle$ for $Y=3$.

columns of Figure 4 we can graphically understand why LABEL classifiers lead to smaller ambiguity: while a CWR has a general region associated with all possible labels, a LABEL classifier more specifically indicates which subsets of the labels are plausible in different areas of the feature space. Clearly, the output of LABEL classifiers is more informative as it assigns only the labels that are deemed plausible in each region.

Unlike classification with reject option, LABEL classification also allows us to control the specific coverage of each class. In the top row and third column of Figure 4 we present the LABEL classification regions when fixing the class-specific coverage at $98 \%$. Although this approach guarantees the desired coverage for each class, we obtain a null region despite the large desired coverage due to the fact that class 1 is very well separated. We therefore use the accretive completion algorithm introduced 
in Section 3.2 to expand the prediction regions, which are shown in the top right plot of Figure 4. The ambiguity of the final classifier is 1.135 .

We also explored the performance of a $k \mathrm{NN}$ classifier, with $k=15$ chosen by leave-oneout cross validation. In the bottom row of Figure 4 we present plots analogous to those obtained from the LABEL kernel classifier. We can see that the classification regions under both approaches are similar in high density areas but they are dramatically different in low density areas. The ambiguity of the final LABEL nearest neighbors classifier is 1.204 .

\subsection{Iris data}

The well-known Iris data consists of 50 samples from each of three species of Iris (1: Iris setosa, 2: Iris virginica, and 3: Iris versicolor). There are four features: the length and the width of the sepals and petals, in centimeters. Due to the small sample size we only illustrate the in-sample behavior of our method, without any sample splitting. We use a standard multinomial logistic model as the fitting method. We first construct a LABEL classifier with class-specific coverage of $98 \%$, which leads to class thresholds of $0.999,0.585$, and 0.660 , meaning that some regions of the feature space do not have any assigned class label. We then use accretive completion to eliminate this null region, which decreases the thresholds to $0.376,0.203$, and 0.411 , with a final ambiguity of 1.007 . The final classifier assigns single labels to all sample points except for one, which receives labels 2 and 3. The left panel of Figure 5 shows that this ambiguous instance is indeed on the boundary between classes 2 and 3 .

To analyze these data using a CWR we need to choose $\rho \in[0,2 / 3]$. In this case

all values in that range lead to a CWR with total coverage greater that $98 \%$, and taking $\rho>2 / 3$ leads to a CWR that never outputs $R$. Therefore with these data no 

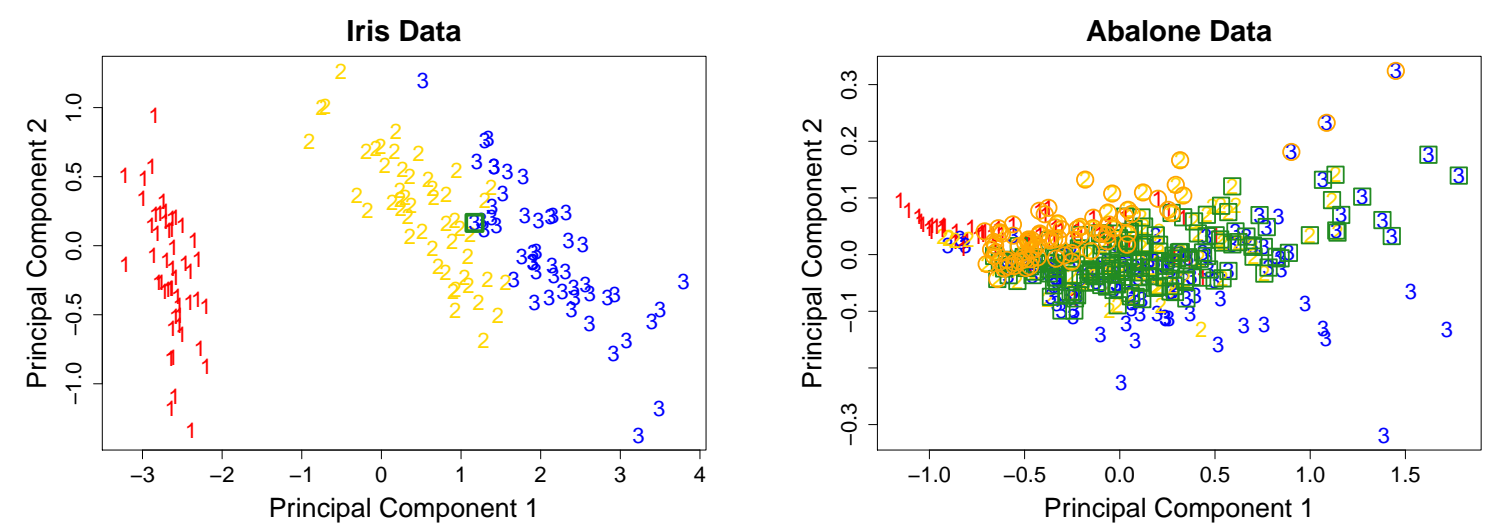

Figure 5: Display of the Iris and Abalone (males) data on their first principal components. Each instance is marked with its true class number. Ambiguous instances labeled with $\{1,2\}$ and $\{2,3\}$ by the LABEL classifier are respectively marked with $\circ$ and $\square$.

comparable CWR leads to identifying any sample point as ambiguous.

\subsection{Abalone data}

The abalone data, available from the UCI Machine Learning Repository (Lichman, 2013), contains measurements of 4177 abalones. The goal is to predict the age from eight other easy-to-obtain measurements including sex, length, diameter, height, weight (whole, meat, gut, shell). To ease the presentation, we grouped the age variable into three categories: 1, young (age 0 to 8); 2, middle (age 9 to 10); 3, old (age 11 and older). We randomly took one fifth of the data as the testing sample and kept the remainder as the training sample. For training we used split-conformal inference with a multinomial logistic regression with lasso penalty tuned by ten-fold cross-validation.

The data exhibits substantial overlap between classes, as shown in the right panel of 
Figure 5. An initial LABEL classifier with a target total coverage of $90 \%$ leads to a threshold of 0.217 , which means that it does not lead to a null region. The absence of null region, despite the low initial coverage requirement, is due to the heavy overlap of the classes. The test-sample class-specific coverages are 89\%, 90.7\%, and $92.1 \%$, with ambiguity of 1.78 .

A CWR with $90 \%$ total coverage is obtained with $\rho=0.4$, and it leads to test-sample class-specific coverages of $95.5 \%, 78 \%$, and $93.9 \%$, with ambiguity of 2.02. Again, the ambiguity of this classifier is larger than the LABEL classifier, given that a CWR always outputs all labels rather than only the plausible labels for each ambiguous instance.

This example clearly illustrates that controlling total coverage, whether in a LABEL classifier or in a CWR, can lead to great imbalance in the coverage of each class. We then obtain a LABEL classifier that controls each class-specific coverage at 90\%, obtaining thresholds of $0.21,0.22$, and 0.22 , meaning that this classifier does not have a null region, again due to the heavy overlap between the classes. In the right panel of Figure 5 we illustrate the results of our final LABEL classifier using the first two principal components of the numeric features of male abalones. We can see that many instances are ambiguous as the classifier has difficulty distinguishing between young and middle abalones, and between middle and old abalones. The classifier however does not mix young and old abalones. The test-sample ambiguity of this final classifier is 1.77 .

\subsection{Zip code data}

We now analyze the zip code data (Le Cun et al., 1990), where the training sample contains 7291 gray scale $16 \times 16$ images of hand-written digits, and the class labels 
correspond to one of the ten digits from "0" to "9". The class labels are relatively balanced, with the most frequent digit " 0 " having a proportion of $16.8 \%$ and the least frequent digit "8" having 7.6\%. The test sample has 2007 images. The zip code data has been analyzed using a similar framework in Lei (2014), but there the problem is converted to a binary one in an ad hoc manner. Here we treat it much more naturally as a multiclass problem and reveal some interesting features.

We start from a $k \mathrm{NN}$ classifier with $k=10$ and generate an initial LABEL classifier with target class-specific coverage level of 0.95 . We use the split-conformal method described in Section 4.3, using two thirds of the training sample to fit the classifier, and the remaining one third to find the thresholds $\widehat{t}_{y}$. The top row of Figure 6 gives some typical examples of the ambiguous images in the testing sample using this initial classifier. Some of the instances in the test sample are such that $\widehat{\mathbf{H}}(x)=\emptyset$, given that the thresholds of this initial classifier add up to more than one. We therefore used accretive completion to remove this null region. The bottom row of Figure 6 gives examples of images that are marked with multiple labels only after accretive completion. We can see that many of these images are truly ambiguous, even to the human eye, indicating that filling-in the null region with single label assignments can be potentially misleading.

The test-sample ambiguity of the final LABEL classifier is 1.27. In Table 1 we present a matrix with the co-occurrence of labels in the test sample according to the final LABEL classifier. This matrix indicates, for example, that the digits " 3 " and "5", "3" and "8", "4" and "9", and "5" and " 8 " are often hard to tell from each other. It also tells us that it is easy to tell apart " 0 " from "7", "6" from "7", "6" from "9", among others, given that no instances receive these label pairs.

Our final LABEL classifier achieves a test-sample coverage of 0.98 , and therefore we 

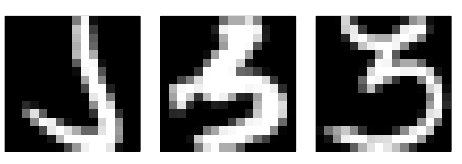

(a) "3" and "5".
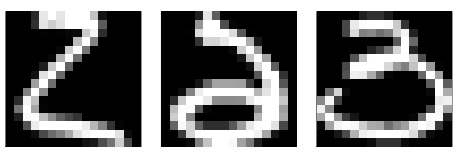

(d) "0", "2" and "3".
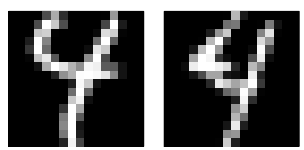

(b) "4" and "9".
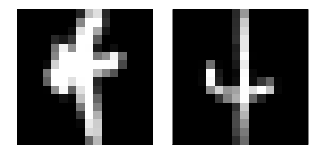

(e) "1" and " 4 ".

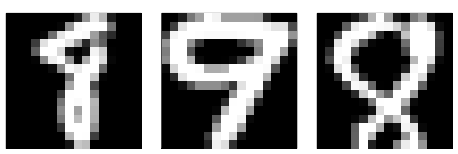

(c) "7", "8" and "9".

Figure 6: Panels (a)-(c): ambiguous images in initial classifier. Panels (d)-(f): additional ambiguous images obtained after applying the accretive completion algorithm.

\begin{tabular}{|c|c|c|c|c|c|c|c|c|c|c|}
\hline \multicolumn{11}{|c|}{ Co-occurrence of Label Assignments } \\
\hline & $" 0 "$ & $" 1 "$ & $" 2 "$ & "3" & "4" & $" 5 "$ & $" 6 "$ & "ry" & "8" & $" 9 "$ \\
\hline$" 0 "$ & 396 & 3 & 36 & 30 & 8 & 35 & 13 & 0 & 13 & 1 \\
\hline$" 1 "$ & 3 & 312 & 9 & 4 & 34 & 7 & 4 & 0 & 10 & 2 \\
\hline "2" & 36 & 9 & 243 & 35 & 20 & 26 & 0 & 10 & 32 & 1 \\
\hline "3" & 30 & 4 & 35 & 305 & 7 & 109 & 0 & 4 & 70 & 2 \\
\hline "4" & 8 & 34 & 20 & 7 & 268 & 9 & 4 & 9 & 14 & 55 \\
\hline$" 5 "$ & 35 & 7 & 26 & 109 & 9 & 234 & 9 & 3 & 43 & 3 \\
\hline$" 6 "$ & 13 & 4 & 0 & 0 & 4 & 9 & 171 & 0 & 1 & 0 \\
\hline "ry" & 0 & 0 & 10 & 4 & 9 & 3 & 0 & 192 & 5 & 15 \\
\hline$" 8 "$ & 13 & 10 & 32 & 70 & 14 & 43 & 1 & 5 & 214 & 8 \\
\hline$" 9 "$ & 1 & 2 & 1 & 2 & 55 & 3 & 0 & 15 & 8 & 218 \\
\hline
\end{tabular}

Table 1: Summary results of applying the LABEL classifier to the zip code data. 
build a CWR that achieves the same total coverage. The CWR that we obtain has a test-sample ambiguity of 2.09. Similarly as in the previous examples, a CWR leads to a higher ambiguity given that all of its ambiguous outputs have cardinality 10, whereas our LABEL classifiers are more specific providing sets of plausible labels.

\section{Discussion}

LABEL classifiers are the least ambiguous set-valued classifiers that guarantee certain prediction confidence levels. This framework for classification builds on the strengths of traditional single-valued classifiers, but provides a more informative and principled approach when dealing with ambiguous instances. As illustrated in Section 5 and in the simulation studies presented in the Supplementary Materials, we find that LABEL classification provides us with more informative outcomes when compared with classification with reject option, as the former outputs class labels that are plausible for each instance rather than a generic reject option. LABEL classifiers also give us more control on the desired coverage requirements, as one can control total or class-specific confidence levels. LABEL classifiers can sometimes output prediction sets that are empty, but we provided different remedies for this problem.

There are many issues that deserve further investigation. An important question is how can our consistency results (Theorem 14) be adapted to the modified classifiers obtained from the accretive completion procedure. In terms of possible extensions, perhaps the most important one is how to deal with a large number of classes. In this regard, a promising approach is to organize the classes into a structure such as a tree. The tree could be based on prior knowledge or be discovered from the data.

Finally, we note a possible further enhancement of our method for class discovery, the 
idea being to look for new classes in the data. For example, if we find well defined clusters in a zone where there is either high ambiguity or null predictions, then these observations could potentially correspond to a new class.

\section{Supplementary Materials}

The online supplementary materials contain the proofs of our theoretical results, $\mathrm{R}$ code to reproduce the examples in Section 5 , and additional simulation studies.

\section{References}

Audibert, J.-Y. and Tsybakov, A. B. (2007). Fast learning rates for plug-in classifiers. The Annals of Statistics, 35:608-633.

Bartlett, P. L. and Wegkamp, M. H. (2008). Classification with a reject option using a hinge loss. The Journal of Machine Learning Research, 9:1823-1840.

Chow, C. K. (1970). On optimum recognition error and reject tradeoff. IEEE Transactions on Information Theory, 16:41-46.

del Coz, J. J., Diez, J., and Bahamonde, A. (2009). Learning nondeterministic classifiers. The Journal of Machine Learning Research, 10:2273-2293.

Denis, C. and Hebiri, M. (2015). Consistency of plug-in confidence sets for classification in semi-supervised learning. arXiv:1507.07235.

Devroye, L. (1978). The uniform convergence of nearest neighbor regression function estimators and their application in optimization. IEEE Transactions on Information Theory, 24(2):142-151. 
Grycko, E. (1993). Classification with set-valued decision functions. In Opitz, O., Lausen, B., and Klar, R., editors, Information and Classification, Studies in Classification, Data Analysis and Knowledge Organization, pages 218-224. Springer Berlin Heidelberg.

Herbei, R. and Wegkamp, M. H. (2006). Classification with Reject Option. Canadian Journal of Statistics, 34(4):709-721.

Le Cun, Y., Boser, B., Denker, J. S., Henderson, D., Howard, R. E., Hubbard, W., and Jackel, L. D. (1990). Handwritten digit recognition with a back-propagation network. In Touretzky, D. S., editor, Advances in Neural Information Processing Systems 2. Proceedings of the 1989 Conference, pages 396-404. Morgan Kaufmann.

Lei, J. (2014). Classification with confidence. Biometrika, 101(4):755-769.

Lei, J., Rinaldo, A., and Wasserman, L. (2014). A conformal prediction approach to explore functional data. Annals of Mathematics and Artificial Intelligence, page in press.

Lei, J., Robins, J., and Wasserman, L. (2013). Distribution free prediction set. Journal of the American Statistical Association, 108:278-287.

Lei, J. and Wasserman, L. (2014). Distribution free prediction bands for nonparametric regression. Journal of the Royal Statistical Society, Series B, 76:71-96.

Lichman, M. (2013). UCI machine learning repository.

Papadopoulos, H., Proedrou, K., Vovk, V., and Gammerman, A. (2002). Inductive confidence machines for regression. In Machine Learning: ECML 2002, pages 345-356. Springer. 
Ramaswamy, H. G., Tewari, A., and Agarwal, S. (2015). Consistent algorithms for multiclass classification with a reject option. arXiv preprint arXiv:1505.0413\%.

Shafer, G. and Vovk, V. (2008). A tutorial on conformal prediction. Journal of Machine Learning Research, 9:371-421.

Silverman, B. W. (1986). Density estimation for statistics and data analysis, volume 26. CRC press.

Stone, C. (1982). Optimal global rates of convergence for nonparametric regression. The Annals of Statistics, 10:1040-1053.

Tsoumakas, G. and Katakis, I. (2007). Multi-label classification: an overview. International Journal of Data Warehousing \&3 Mining, 3(1):1-13.

Tsybakov, A. B. (2009). Introduction to Nonparametric Estimation. Springer, New York.

van de Geer, S. A. (2008). High-dimensional generalized linear models and the lasso. The Annals of Statistics, 36:614-645.

Vovk, V. (2013). Conditional validity of inductive conformal predictors. Machine Learning, 92:349-376.

Vovk, V., Fedorova, V., Nouretdinov, I., and Gammerman, A. (2016). Criteria of efficiency for conformal prediction. In Proceedings of COPA 2016 (Fifth Symposium on Conformal and Probabilistic Prediction with Applications), pages 23-39.

Vovk, V., Gammerman, A., and Shafer, G. (2005). Algorithmic Learning in a Random World. Springer, New York.

Vovk, V., Petej, I., and Fedorova, V. (2014). From conformal to probabilistic 
prediction. In COPA 2014 Proceedings, Artificial Intelligence Applications and Innovations, pages 221-230.

Yuan, M. and Wegkamp, M. (2010). Classification methods with reject option based on convex risk minimization. Journal of Machine Learning Research, 11:111-130.

Zhang, M. L. and Zhou, Z. H. (2007). Ml-knn: A lazy learning approach to multi-label learning. Pattern Recognition, 40(7):2038-2048. 


\section{Supplementary Materials for "Least Ambiguous Set- Valued Classifiers with Bounded Error Levels"}

\section{A Proofs}

We present the proofs of the results that are not clear in the main article.

Lemma 5. Proof. Let $\mathbf{H}$ and $\mathbf{H}^{\prime}$ be such that $\mathbb{P}\{Y \in \mathbf{H}(X) \mid Y=y\}=\mathbb{P}\{Y \in$ $\left.\mathbf{H}^{\prime}(X) \mid Y=y\right\}=1-\alpha_{y}$, and $\mathbb{P}\left\{y \in \mathbf{H}^{\prime}(X) \mid Y \neq y\right\} \geq \mathbb{P}\{y \in \mathbf{H}(X) \mid Y \neq y\}$, for all $y$. Multiplying this expression by $\mathbb{P}(Y \neq y)$ we obtain $\mathbb{P}\left\{y \in \mathbf{H}^{\prime}(X), Y \neq y\right\} \geq \mathbb{P}\{y \in$ $\mathbf{H}(X), Y \neq y\}$, which can be rewritten as

$$
\sum_{l \neq y} \mathbb{P}\left\{y \in \mathbf{H}^{\prime}(X) \mid Y=l\right\} \pi_{l} \geq \sum_{l \neq y} \mathbb{P}\{y \in \mathbf{H}(X) \mid Y=l\} \pi_{l},
$$

which holds for all $y$. On the other hand we have

$$
\sum_{y} \mathbb{P}\left\{Y \in \mathbf{H}^{\prime}(X) \mid Y=y\right\} \pi_{y}=\sum_{y} \mathbb{P}\{Y \in \mathbf{H}(X) \mid Y=y\} \pi_{y}=\sum_{y}\left(1-\alpha_{y}\right) \pi_{y} .
$$

Adding Expression (9) over all $y$ and combining with the last expression leads to

$$
\sum_{y} \sum_{l} \mathbb{P}\left\{y \in \mathbf{H}^{\prime}(X) \mid Y=l\right\} \pi_{l} \geq \sum_{y} \sum_{l} \mathbb{P}\{y \in \mathbf{H}(X) \mid Y=l\} \pi_{l},
$$

which by the law of total probability and Remark 4 is equivalent to $\mathbb{E}\left\{\left|\mathbf{H}^{\prime}(X)\right|\right\} \geq$ $\mathbb{E}\{|\mathbf{H}(X)|\}$.

Theorem 6. Proof. First, notice that $\operatorname{logit}\{p(y \mid x)\}=\log \left\{p(x \mid y) / p\left(x \mid y^{c}\right)\right\}+$ $\operatorname{logit}\left(\pi_{y}\right)$, where $p\left(x \mid y^{c}\right) \equiv \sum_{j \neq y} p(x \mid Y=j) \pi_{j} / \sum_{j \neq y} \pi_{j}$. Given that the $\log$ and logit functions are monotonically increasing, this expression implies that the decision regions $C_{y}$ can alternatively be based on level sets of the likelihood ratios 
$\Lambda_{y}(x)=p(x \mid y) / p\left(x \mid y^{c}\right)$, that is $C_{y}=\left\{x: \Lambda_{y}(x) \geq \ell_{y}\right\}$ with $\ell_{y}$ chosen so that $\mathbb{P}\left(C_{y} \mid Y=y\right)=1-\alpha_{y}$. The region $C_{y}^{c}$ therefore corresponds to the Neyman-Pearson rejection region for testing the null hypothesis $H_{0}: Y=y$ versus $H_{1}: Y \neq y$. By the Neyman-Pearson lemma we have that the classifier $\mathbf{H}$ induced by the sets $C_{y}$ maximizes the probabilities $\mathbb{P}\{y \notin \mathbf{H}(X) \mid Y \neq y\}$, or equivalently $\mathbb{P}\{y \in \mathbf{H}(X) \mid Y \neq y\}$ is minimized. Finally, by Lemma 5 we have that this decision rule $\mathbf{H}$ also minimizes the ambiguity.

Theorem 8. Proof. Firstly, since $\widetilde{\mathbb{A}}$ is the optimal value of problem $(3), \widetilde{\mathbb{A}} \leq$ $\mathbb{A}\left(\mathbf{H}^{\dagger}\right)$. Now, $\mathbb{A}\left(\mathbf{H}^{\dagger}\right)=\mathbb{E}\left[I\left\{X \in \mathcal{N}\left(\mathbf{H}^{*}\right)\right\}\left|\mathbf{H}^{\dagger}(X)\right|\right]+\mathbb{E}\left[I\left\{X \notin \mathcal{N}\left(\mathbf{H}^{*}\right)\right\}\left|\mathbf{H}^{\dagger}(X)\right|\right]=$ $\mathbb{P}\left\{\mathcal{N}\left(\mathbf{H}^{*}\right)\right\}+\mathbb{A}\left(\mathbf{H}^{*}\right)$, and the result follows from $\mathbb{A}\left(\mathbf{H}^{*}\right) \leq \widetilde{\mathbb{A}}$ given that (2) is a relaxation of (3).

Theorem 14. Proof. The first part is essentially the same as in Lei (2014). We prove the second part. Let $\widehat{G}_{y}$ be the empirical distribution of $p\left(y \mid X_{y, 1}\right), \ldots, p\left(y \mid X_{y, n_{y}}\right)$ where $X_{y, 1}, \ldots, X_{y, n_{y}}$ are sample points in class $y$. Let $\widehat{\mathbb{P}}_{y}(\cdot)$ be the probability measure corresponding to $\widehat{G}_{y}$. Define $L_{y}(t)=\{x: p(y \mid x) \leq t\}, \widehat{L}_{y}(t)=\{x: \widehat{p}(y \mid x) \leq t\}$.

We focus on the event

$$
\begin{gathered}
E=\left\{\sup _{y, x}|\widehat{p}(y \mid x)-p(y \mid x)| \leq \epsilon_{n}, \sup _{y, t}\left|\widehat{G}_{y}(t)-G_{y}(t)\right| \leq c \sqrt{\frac{\log n}{n}},\right. \\
\left.\sup _{y}\left|\widehat{\pi}_{y}-\pi_{y}\right| \leq c \sqrt{\frac{\log n}{n}}\right\}
\end{gathered}
$$

which has probability at least $1-K \delta_{n}-n^{-1}$ if $c$ is chosen large enough and $K$ grows slowly with $n$. Here the first inequality in $E$ is given by our assumption in (4) and the other two follow from standard empirical process theory.

Recall that for total coverage we use the same threshold for all classes. Let $t^{*}=G^{-1}(\alpha)$ be the ideal cut-off value for $p(y \mid x)$. If $t \leq t^{*}-\epsilon_{n}-\left\{(K+1) c c_{1}^{-1} \sqrt{\log n / n}\right\}^{1 / \gamma}$, then 
we have

$$
\begin{aligned}
\widehat{\mathbb{P}}_{y}\left\{\widehat{L}_{y}(t)\right\} & \leq \widehat{\mathbb{P}}_{y}\left\{L\left(t+\epsilon_{n}\right)\right\}=\widehat{G}_{y}\left(t+\epsilon_{n}\right) \leq G_{y}\left(t+\epsilon_{n}\right)+c \sqrt{\frac{\log n}{n}} \\
& \leq G_{y}\left[t^{*}-\left\{(K+1) c c_{1}^{-1} \sqrt{\log n / n}\right\}^{1 / \gamma}\right]+c \sqrt{\frac{\log n}{n}} \leq G_{y}\left(t^{*}\right)-c K \sqrt{\frac{\log n}{n}} .
\end{aligned}
$$

Therefore,

$$
\widehat{t}>t^{*}-\epsilon_{n}-\left\{(K+1) c c_{1}^{-1} \sqrt{\log n / n}\right\}^{1 / \gamma},
$$

because otherwise we have

$$
\begin{aligned}
\sum_{y=1}^{K} \widehat{\pi}_{y} \widehat{\mathbb{P}}_{y}\left\{\widehat{L}_{y}(\widehat{t})\right\} & \leq \sum_{y=1}^{K} \widehat{\pi}_{y}\left\{G_{y}\left(t^{*}\right)-c K \sqrt{\log n / n}\right\} \\
& \leq \alpha+\sum_{y=1}^{K}\left|\widehat{\pi}_{y}-\pi_{y}\right| G_{y}\left(t^{*}\right)-c K \sqrt{\log n / n}<\alpha .
\end{aligned}
$$

Similarly we can obtain

$$
\widehat{t} \leq t^{*}+\epsilon_{n}+\left\{(K+1) c c_{1}^{-1} \sqrt{\log n / n}\right\}^{1 / \gamma},
$$

and combining 10 and 11 we have $\left|\widehat{t}-t^{*}\right| \leq \epsilon_{n}+\left\{(K+1) c c_{1}^{-1} \sqrt{\log n / n}\right\}^{1 / \gamma}$. (It is worth noting that a rigorous argument of this would require $\widehat{p}(y \mid x)$ to have distinct values at the sample points $X_{1}, \ldots, X_{n}$. This is a minor issue because one can always add very small random perturbations such as $\widehat{p}(y \mid X)+\xi$ with $\xi \sim$ $\operatorname{Unif}\left(-n^{-2}, n^{-2}\right)$.)

Then

$$
\begin{aligned}
\mathbb{P}_{y}\left(\widehat{C}_{y} \backslash C_{y}^{*}\right) & =\mathbb{P}_{y}\left\{\widehat{p}(y \mid X) \geq \widehat{t}, p(y \mid X)<t^{*}\right\} \\
& \leq \mathbb{P}_{y}\left[t^{*}-2 \epsilon_{n}-\left\{(K+1) c c_{1}^{-1} \sqrt{\log n / n}\right\}^{1 / \gamma} \leq p(y \mid X)<t^{*}\right] \\
& \leq c^{\prime}\left(\epsilon_{n}^{\gamma}+K \sqrt{\log n / n}\right),
\end{aligned}
$$


for some constant $c^{\prime}$ depending on $c, c_{1}, \gamma$. Similarly we can obtain $\mathbb{P}_{y}\left(C_{y}^{*} \backslash \widehat{C}_{y}\right) \leq$ $c^{\prime}\left(\epsilon_{n}^{\gamma}+K \sqrt{\log n / n}\right)$, and hence $\mathbb{P}_{y}\left(\widehat{C}_{y} \triangle C_{y}^{*}\right) \leq c^{\prime}\left(\epsilon_{n}^{\gamma}+K \sqrt{\log n / n}\right)$. Summing over $y$ we have

$$
\mathbb{P}\left(\widehat{\mathbf{H}} \triangle \mathbf{H}^{*}\right)=\sum_{y=1}^{K} \pi_{y} \mathbb{P}_{y}\left(\widehat{C}_{y} \triangle C_{y}^{*}\right) \leq c^{\prime}\left(\epsilon_{n}^{\gamma}+K \sqrt{\log n / n}\right) .
$$

\section{B Simulation Studies}

\section{B.1 Univariate Scenarios}

We start with a simple setting to illustrate the fundamental differences between classifiers with reject option (CWRs) and LABEL classifiers. In this comparison we simulate samples of size $n=4000$, drawing $Y$ from $\{1,2,3\}$ with probabilities that change in three simulation scenarios, summarized in Table 2. We take $X$ to be univariate with distributions $(X \mid Y=y)$ being normal with means $-2,0$, and 2 , and variances equal to 1 . We estimate $p(y \mid x)$ using $\widehat{p}(y \mid x)=\widehat{p}_{y}(x) \widehat{\pi}_{y} / \sum_{l} \widehat{p}_{l}(x) \widehat{\pi}_{l}$, where $\widehat{\pi}_{y}=\sum_{i} I\left(Y_{i}=y\right) / n$ and $\widehat{p}_{y}(x)$ being a Gaussian density. We use total coverage of 0.95 for the plug-in LABEL and CWR classifiers. For each scenario we repeat the simulation 1000 times, and in Table 2 we report the average ambiguity and class coverages across simulations.

From Table 2 we can see that, across the three scenarios that we considered, the LABEL classifier has smaller average ambiguity than the CWR. In fact, the ambiguity of the LABEL classifier was smaller in all 1000 simulation replicates within each simulation scenario, not only on average. This is rather natural, since in our construction of LABEL classifiers assigning the output $\{1,2,3\}$ to a sample point is penalized 


\begin{tabular}{cccccc}
\hline & \multicolumn{2}{c}{ CWR } & & \multicolumn{2}{c}{ LABEL } \\
\cline { 2 - 3 } \cline { 5 - 6 } Class Probs. & Ambiguity & Class Coverage & & Ambiguity & Class Coverage \\
\hline$(.45, .10, .45)$ & 1.28 & $(1.00,0.53,1.00)$ & & 1.21 & $(0.98,0.64,0.98)$ \\
$(.33, .33, .34)$ & 1.94 & $(0.98,0.89,0.98)$ & & 1.51 & $(0.96,0.93,0.96)$ \\
$(.60, .30, .10)$ & 1.78 & $(0.99,0.89,0.92)$ & & 1.41 & $(0.98,0.91,0.88)$ \\
\hline
\end{tabular}

Table 2: Comparing LABEL classifiers with total coverage control to CWRs in a three-class problem. Simulation details are given in the main text. Quantities reported here are averages over 1000 simulation replicates.

more than an output containing only two labels. CWRs effectively assign the output $\{1,2,3\}$ to all ambiguous sample points, and therefore do not specify which labels are plausible for a given instance. In the scenarios explored in our simulation study, classes 1 and 3 are relatively well separated, and therefore the LABEL classifier assigns the outcomes $\{1,2\}$ and $\{2,3\}$ to the sample points in the overlap of classes 1 and 2 , and 2 and 3 , respectively. To such cases, the CWR assigns $\{1,2,3\}$, which is less informative and leads to larger ambiguity. LABEL classifiers are therefore more informative in reporting ambiguous cases as they indicate the set of plausible labels for each instance.

We also conclude from Table 2 that controlling total coverage can lead to very uneven class coverage with both LABEL classifiers and CWRs. Notwithstanding, our framework can also be used to control class-specific coverage, something that cannot be done using CWRs. 


\begin{tabular}{ccc}
\hline & Ambiguity & Class Coverage \\
\hline CWR & 3.64 & $(0.87,0.98,0.93,0.92,0.97)$ \\
LABEL & 1.87 & $(0.90,0.99,0.97,0.69,0.53)$ \\
\hline
\end{tabular}

Table 3: Comparing LABEL classifiers and CWRs under total coverage control in a multivariate problem. Simulation details are given in the main text. Quantities reported here are averages over 1000 simulation replicates.

\section{B.2 Data-Based Multivariate Scenario}

To explore the performance of LABEL classifiers in comparison with CWRs under more complex scenarios, we use the Abalone data analyzed in Section 5.4 to create a synthetic population from which we simulate. The goal here is to construct a synthetic population based on real data, not necessarily study the repeated sample performance of the analysis in Section 5.4 . We start by creating $K=5$ classes using the age variable (unlike in Section 5.4, where we took $K=3$ to ease the presentation). We then use the seven numeric features in the Abalone data to obtain a mean vector and a covariance matrix within each class. The population is then defined as a five-component mixture of seven-dimensional normal distributions, with means, covariance matrices, and population proportions obtained from the abalone data.

Each of the 1000 replicates in this simulation study consists of $n=4000$ draws from the aforementioned mixture. The estimator of $p(y \mid x)$ is a simple multinomial logistic regression. We controlled the total coverage of the classifiers at 0.95 . For CWRs this means that we chose the cost of the reject option $\rho$ so that its total coverage is greater or equal to 0.95 .

In Table 3 we compare the ambiguity and class coverages that we obtain on average 


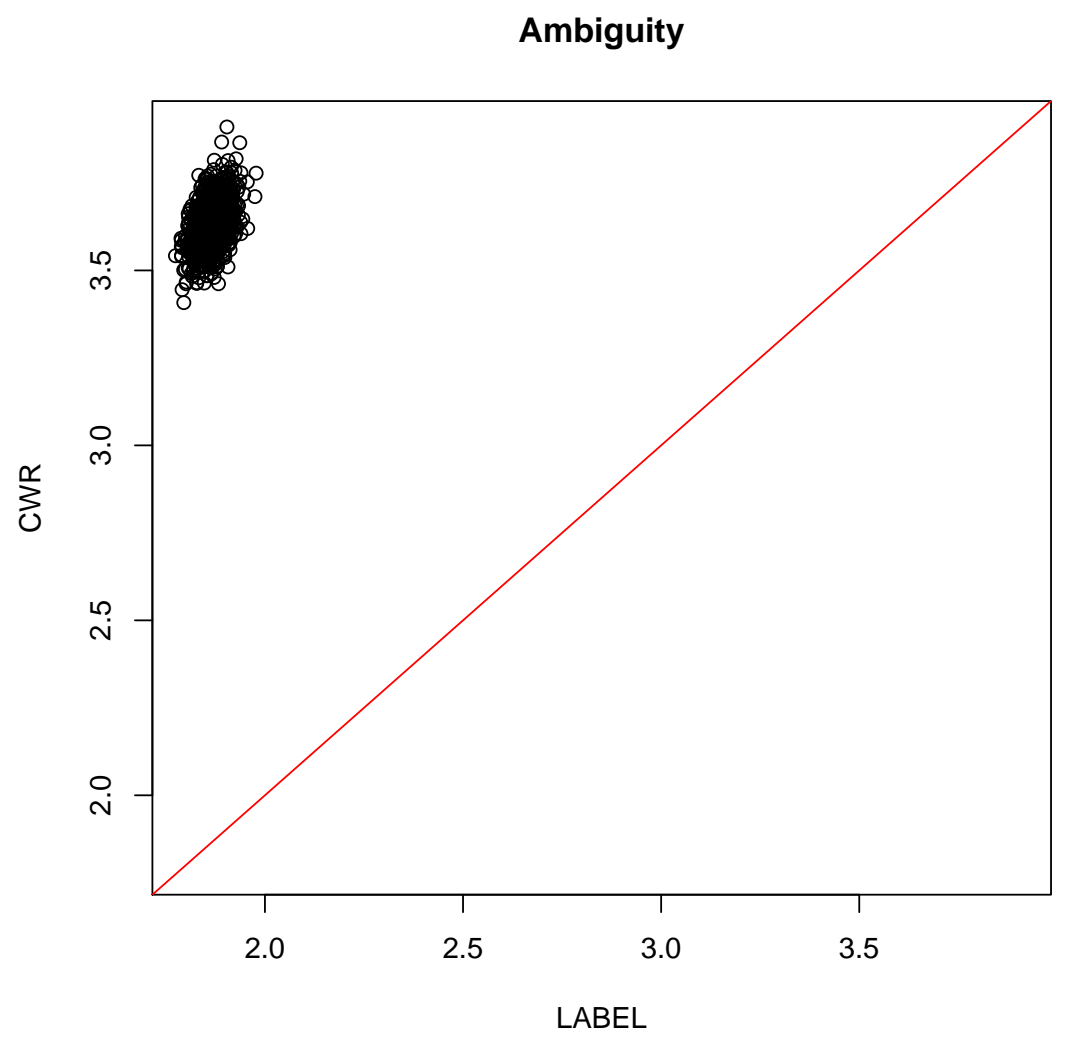

Figure 7: Comparison of ambiguity obtained from LABEL and CWR across 1000 simulation replicates in multivariate scenario.

from LABEL and CWR. Similarly as with the other examples and simulation scenarios, LABEL classifiers provide smaller ambiguity values, meaning that these are more informative than CWRs as the latter provide larger outputs. Indeed, in Figure 7 we show that LABEL ambiguity is smaller than CWR ambiguity in all simulation replicates.

In Table 3 we can also see that LABEL classifiers tend to give very imbalanced class coverages. In this simulation scenario this occurs because three of the classes have 
very small class probabilities (classes 1,4 and 5), and in addition two of these are not very well separated from a third one (classes 4 and 5 are very close to class 3 ). This phenomenon was illustrated in Example 3. Regarding CWRs, their larger outputs lead in this case to higher and more balanced class coverages given that instances receive all labels when they get assigned the reject option. Nevertheless, balance of class coverage cannot be guaranteed with CWRs, whereas LABEL classifiers can indeed be used under class-specific coverage control. 